\title{
Otimização da modelação de remoção de nitrogênio em sistemas de lodo ativado
}

\section{Modelling of nitrogen removal optimization in activated sludge systems}

Data de entrada: 05/12/2016

Data de aprovação: $10 / 02 / 2017$

\section{Resumo}

A remoção de nitrogênio tem sido tradicionalmente realizada em sistemas de lodo ativado de fluxo contínuo (FC), mas recentemente a remoção em sistemas de lodos ativados operando sob um regime de alimentação em bateladas sequenciais (RBS) tem aplicação crescente. Neste trabalho se desenvolve um modelo de nitrificação e desnitrificação no sistema de lodo ativado e se comparam os aspectos mais importantes de quatro variantes do sistema em termos da capacidade de remoção de nitrogênio. Mostra-se que a extensão máxima da remoção de nitrogênio é determinada pelas características do esgoto e por constantes cinéticas, mas num grau importante, também pelo tipo de sistema de remoção de nitrogênio (FC ou RBS) e pela natureza do lodo (floculento ou granulado). O sistema RBS com nitrificação e desnitrificação simultânea num reator unitário com lodo aeróbio granular mostrou ter a maior capacidade de remoção de nitrogênio. $O$ sistema RBS com reatores dedicados para nitrificação e desnitrificação é o melhor quando se usa lodo floculento.

Palavras-chave: Remoção de nitrogênio. Modelação. Otimização.

\section{Abstract}

Nitrogen removal in activated sludges systems has been traditionally accomplished in continuous flow (CF) systems, but recently nitrogen removal in systems operated in sequential batch mode (SBR) has increasing application. In this paper a model for nitrification and denitrifications is presented and the most important aspects of nitrogen removal in four variants of the system are compared in terms of their nitrogen removal capacity. It is shown that the maximum extent of nitrogen removal is determined not only by the sewage characteristics and kinetic constants of the relevant biological processes, but to the large degree also depends on the nitrogen removal system (CF or SBR) and the nature of the sludge (flocculent or granular). The SBR variant with simultaneous nitrification and denitrification in a single reactor with granular sludges has shown to have the biggest nitrogen removal capacity. The SBR system with dedicated zones for nitrification and denitrification is the best solution when flocculent sludge is used. Keywords: Nitrogen removal. Modelling. Optimization.

Heraldo Antunes Silva Filho* - Doutor em Engenharia Ambiental pela Universidade Estadual da Paraíba (UEPB). Professor do Instituto Federal de Educação, Ciência e Tecnologia do Ceará (IFCE) E-mail: heraldoifceagmail.com.

Adrianus van Haandel - PhD em Engenharia Civil na University Of Cape Town - África do Sul. Professor da Universidade Federal de Campina Grande (UFCG). E-mail: adrianusvhagmail.com.

Lincoln Soares Mota - Mestre em Engenharia Ambiental pela Universidade Estadual da Paraíba (UEPB). Engenheiro profissional. E-mail: lincoln_ smahotmail.com

"Endereço para correspondência: Instituto Federal de Educação, Ciência e Tecnologia do Ceará (IFCE), Campus Limoeiro do Norte, Eixo de Ambiente e Saúde. CEP: 62930-000,Limoeiro do Norte, Ceará. Telefone: (88) 3447-6413. 


\section{INTRODUÇÃO}

Logo depois de ficarem estabelecidos os conceitos básicos da nitrificação (DOWNING et al 1964) e desnitrificação (LUDZACK e ETTINGER, 1964 e WUHRMANN, 1964), os sistemas de lodo ativado para a remoção biológica de nitrogênio foram desenvolvidos. Esses sistemas tradicionais como as configurações Bardenpho (BARNARD, 1976) e UCT (SIEBRITZ et al. 1982) eram de fluxo contínuo e tinham uma série de reatores, seguido por um decantador final. A Figura 1 é uma representação esquemática de um sistema Bardenpho, com três reatores e um decantador final com o afluente passando sequencialmente por esses reatores. No reator aeróbio, bactérias autotróficas realizam a nitrificação, a oxidação de amônia para nitrato. Nos reatores anóxicos, a desnitrificação se desenvolve: o nitrato é reduzido a nitrogênio molecular e o material orgânico é oxidado por bactérias heterotróficas.

Nitrificação e Desnitrificação Simultânea (NDS) com aeração limitada em um único reator também foi experimentada em sistemas FC de remoção de nitrogênio (MATSCHE, 1972), mas foi abandonada porque os resultados tendiam a ser instáveis. Mais recentemente, tal prática tem encontrado aplicação renovada em sistemas de RBS (RITTMAN, 1985; HELLINGA et al., 1998; POCHANA et al., 1999; SATOH et al., 2003), tendo-se duas configurações básicas: (1) O sistema NDS com um único reator onde ocorrem os dois processos; e (2) O sistema $\mathrm{N}$-D composto por duas zonas, uma aeróbia e outra anóxica, com recirculação interna para transferir o nitrato produzido na zona aeróbia para a zona anóxica. Na Figura 2 se apresenta um esquema dessas duas alternativas. As duas variantes são extremas: é possível ter um único reator com zonas de uma concentração de oxigênio dissolvido relativamente elevada, nas quais a nitrificação predomina e zonas com uma concentração baixa de oxigênio dissolvido (OD), onde prevalece a desnitrificação.

Para um desempenho ótimo de ambos os sistemas, é necessário que a produção de nitrato seja igual à remoção de nitrato, de modo que, no final do tratamento de uma batelada, as concentrações tanto de amônia como de nitrato sejam baixas. Se esse não for o caso, haverá nitrogênio no efluente, seja na forma de $\mathrm{NH}_{4}{ }^{+}$ou na forma de $\mathrm{NO}_{3}{ }^{-}$. No sistema NDS a concentração de OD é usada para o controle da remoção de nitrogênio: quanto menor a concentração de OD, menor é a taxa de nitrificação e maior a taxa de desnitrificação. Para a concentração ótima de OD, as taxas de ambos os processos são iguais durante o tratamento.

No sistema $N-D$, a divisão da massa de lodo nas zonas aeróbia e anóxica é a variável de controle. As massas de lodo na zona aeróbia e anóxico são estabelecidas de tal modo que a capacidade de nitrificação na zona aeróbia seja igual à capacidade de desnitrificação da zona anóxica. A divisão da massa de lodo pode ser variável, alterando-se a fração do volume total do sistema ocupado pelas duas zonas: se o nitrato na zona anóxica aumenta, seu volume deve ser aumentado e, consequentemente, o volume da zona aeróbia deve ser reduzido. Para um desempenho ideal desses sistemas, as variáveis mais importantes (OD para NDS e massa de lodo ou volume das zonas para $\mathrm{N}-\mathrm{D}$ ) são controlados por sensores online.

Uma aplicação importante da configuração RBS foi o desenvolvimento de sistemas com lodo granular, em vez de lodo floculento, que tinham sido usados desde o desenvolvimento inicial do sistema de lodo ativado. DE KREUK et al. (2005) mostrou que, em condições apropriadas, um lodo com grânulos macroscópicos se desenvolve com excelentes características de sedimentação e elevada atividade. Dentro desses grânulos a transferência de oxigênio é limitada, de modo que zonas anóxicas ou anaeróbias são formadas na parte central dos grânulos, onde a desnitrificação pode se desenvolver. Na periferia do grânulo a nitrificação se desenvolve, enquanto na maior parte da fase líquida uma concentração relativamente elevada OD de 2 a 2,5 mg.L-1 é aplicada (DE KREUK, 2006). 


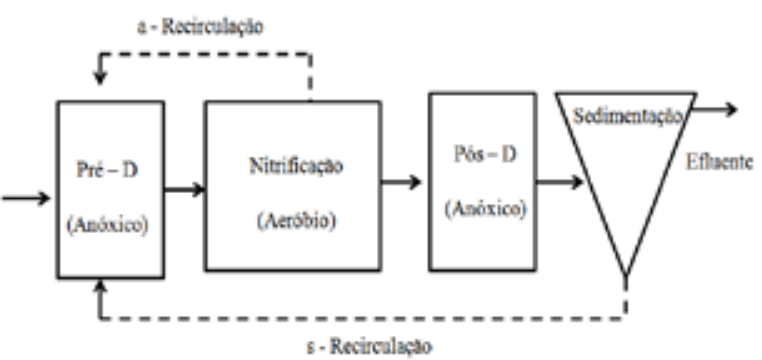

Figura 1: Representação esquemática do sistema Bardenpho para remoção de nitrogênio.

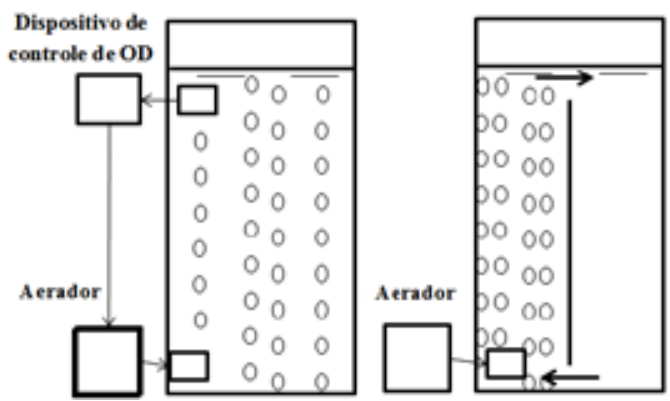

Figura 2: Representação esquemática do sistema NDS (esq.) e N-D (dir.) para remoção de $\mathrm{N}$ em sistemas RBS.

Neste trabalho se mostra que a remoção de nitrogênio em sistemas de lodo ativado pelos processos de nitrificação e desnitrificação pode ser modelada com as mesmas equações básicas, propostas por VAN HAANDEL et al. (1981), embora a capacidade de remoção de nitrogênio seja diferente para as diferentes configurações.

\subsection{Modelação da remoção de nitrogênio em sistemas de lodo ativado}

A massa de lodo em sistemas de lodo ativado com uma concentração uniforme é a mesma em sistemas de fluxo contínuo e em sistemas RBS operados sob condições comparáveis. Nesses sistemas, expressões para a concentração dos diferentes componentes do lodo foram desenvolvidos por Marais e Ekama (1976) e posteriormente incorporadas aos modelos apresentados pela Associação Internacional da Água, IWA (HENZE et al., 1986 e 1994).
Lodo nitrificante (autotrófico)

$X_{n}=Y_{n} R_{s}\left(1+b_{n}\right) N_{c} / R_{h}$

Lodo ativo (heterotrófico):

$X_{a}=\left(1 f_{\text {us }}-f_{\text {up }}\right) Y_{s}\left(1+b_{h}\right) S_{\text {ta }} / R_{h}$

Lodo endógeno: $X_{e}=\mathrm{fb}_{h} \mathrm{R}_{\mathrm{s}} \mathrm{X}_{\mathrm{a}}$

Lodo inerte: $\quad X_{i}=\left(f_{u p} / f_{c v}\right) S_{\text {ta }}$

Lodo volátil: $\quad X_{v}=X_{a}+X_{e}+X_{i}$

$$
=\left[\left(1-f_{\text {us }}-f_{\text {up }}\right)\left(1+f b_{h} R s\right) Y R_{s} /\right.
$$

$\left.\left(1+b_{h} R_{s}\right)+f_{u p} R_{s} / f_{c v}\right] S_{t a} / R_{h}$

Lodo total: $\quad \mathrm{X}_{\mathrm{t}}=\mathrm{X}_{\mathrm{v}} / \mathrm{F}_{\mathrm{v}}$

Onde:

$\mathrm{X}_{\mathrm{n}}=$ Concentração de lodo nitrificante (bactérias vivas autotróficas);

$\mathrm{X}_{\mathrm{a}}=$ Concentração de lodo ativo (bactérias vivas heterotróficas);

$X_{e}=$ Concentração do resíduo endógeno;

$X_{i}=$ Concentração de lodo inerte (gerado de DQQO afluente não biodegradável e particulada);

$X_{v}=$ Concentração de lodo volátil $\left(=X_{a}+X_{e}+X_{i}\right)$;

$\mathrm{X}_{\mathrm{t}}=$ Concentração de lodo total (volátil + mineral);

$\mathrm{f}_{\text {us }}=$ Fração da DQO afluente que é não biodegradável e solúvel;

$\mathrm{f}_{\text {up }}=$ Fração da DQO afluente que é não biodegradável e particulada;

$\mathrm{f}=$ Fração do lodo ativo decaído que permanece como resíduo (0,2, MARAIS e EKAMA, 1976);

$\mathrm{f}_{\mathrm{cv}}=$ Proporção entre lodo volátil e a DQO (1,5 gDQ.O.g-1SVS - MARAIS e EKAMA, 1976);

$\mathrm{f}_{\mathrm{v}}=$ Razão entre lodo volátil e total;

$b_{h}=$ Constante de decaimento de heterótrofas $\left(=0,24^{*} 1,04^{(t-20)} d^{-1}\right.$ MARAIS e EKAMA, 1976); 
$\mathrm{b}_{\mathrm{n}}=$ Constante de decaimento de autótrofas (= $0,04 * 1,04^{(t-20)} d^{-1}$ MARAIS e EKAMA, 1976);

$Y=$ Coeficiente de rendimento para heterótrofas (0,45 gSVS. ${ }^{-1}$ DOO, MARAIS E EKAMA, 1976);

$Y_{n}=$ Coeficiente de rendimento para autótrofas (0,10 gSVS.g-1DQOO, MARAIS E EKAMA, 1976);

$\mathrm{R}_{\mathrm{s}}=$ Idade de lodo;

$R_{h}=$ Tempo de permanência hidráulico;

$\mathrm{S}_{\mathrm{ta}}=$ Concentração da DQQ do afluente;

$N_{c}=$ Concentração de nitrogênio a ser nitrificado.

O processo de nitrificação geralmente é descrito com a cinética de Monod com dois substratos: amônia e oxigênio dissolvido (STENTRÖM e PODUSHKA, 1981; VAN HAANDEL e MARAIS, 1999). Nesse caso, a taxa de nitrificação pode ser descrita como:

$r_{N}=\left(\mu_{\max } / Y_{n}\right) X_{n}\left(N_{a} /\left(N_{a}+K_{n}\right)\right)\left(O D /\left(O D+k_{o}\right)\right)$

Onde:

$r_{N}=$ taxa de nitrificação $\left(\mathrm{mg} \cdot \mathrm{L}^{-1} \cdot \mathrm{d}^{-1}\right)$;

$\mu_{\max }=$ taxa máxima de crescimento de nitrificantes. $\left(\mathrm{d}^{-1}\right)$;

$\mathrm{N}_{\mathrm{a}}=$ concentração do substrato amônia (substrato 1) (mgN.L-1);

$\mathrm{K}_{\mathrm{n}}=$ constante de meia saturação para amônia $\left(\mathrm{mgN} . \mathrm{L}^{-1}\right)$;

$O D=$ concentração de oxigênio dissolvido (substrato 2$)\left(\mathrm{mg}^{\mathrm{L}} \mathrm{L}^{-1}\right)$;

$\mathrm{k}_{\mathrm{o}}=$ constante de meia saturação para OD $\left(\mathrm{mg} \cdot \mathrm{L}^{-1}\right)$.

Ambas as constantes de meia saturação são pequenas ( $\left.<1 \mathrm{mg} \cdot \mathrm{L}^{-1}\right)$, e em um reator aeróbio operando sob o regime de bateladas sequenciais a concentração de amônia é alta até que quase todo este substrato seja nitrificado de modo que: $\mathrm{N}_{\mathrm{a}} \gg \mathrm{K}_{\mathrm{n}}$ e $\mathrm{N}_{\mathrm{a}} /\left(\mathrm{N}_{\mathrm{a}}+\mathrm{K}_{\mathrm{n}}\right) \approx 1$. Nessas condições a Eq. (7a) se simplifica para:

$r_{N}=\left(\mu_{\max } / Y_{n}\right) X_{n}\left(O D /\left(O D+k_{o}\right)=\left(\mu_{m} / Y_{n}\right) X_{n}\right.$

Se a concentração de OD também é alta, a taxa de nitrificação é máxima e se expressa como:

$r_{N \max }=\left(\mu_{\max } / Y_{n}\right) X_{n}$

A taxa de desnitrificação num reator anóxico depende da natureza e da disponibilidade de material orgânico, bem como da presença de nitrato e de lodo ativo. A taxa máxima de desnitrificação se desenvolve quando tanto o substrato solúvel (facilmente biodegradável) como o particulado (lentamente biodegradável) estão disponíveis. Essa taxa foi modelada como (VAN HAANDEL et al., 1981):

$\mathrm{r}_{\mathrm{Dmax}}=\left(\mathrm{k}_{1}+\mathrm{k}_{2}\right) \mathrm{X}_{\mathrm{a}}$

A Eq (8a) indica que a taxa de desnitrificação é proporcional à concentração de lodo ativo (a massa de bactérias heterotróficas), e que a proporcionalidade depende de dois substratos: o material solúvel $\left(k_{1}\right)$ e o material particulado $\left(k_{2}\right)$. Quando o material solúvel é metabolizado e não mais disponível, a Eq. (8a) se simplifica para:

$r_{D}=k_{2} X_{a}$

Depois de metabolizar também o material lentamente biodegradável, a desnitrificação depende da respiração endógena, ou seja, o próprio lodo se torna substrato:

$r_{D}=k_{3} X_{a}$

Onde:

$r_{D}=$ taxa de desnitrificação no reator anóxico;

$\mathrm{k}_{1}, \mathrm{k}_{2}, \mathrm{k}_{3}=$ constantes de desnitrificação associadas com metabolismo de material facilmente e lentamente biodegradável e respiração endógena respectivamente. Van Haandel et al (1981) pro- 
puseram: $\mathrm{k}_{1}=0,73^{*} 1,2^{(\mathrm{t}-20)} ; \mathrm{k}_{2}=0,1^{* 1} 1,08^{(\mathrm{t}-20)} ; \mathrm{k}_{3}=$ $0,08^{* 1} 1,03^{(t-20)} \mathrm{mgN} \cdot \mathrm{mg}^{-1} \mathrm{Xa} \cdot \mathrm{d}^{-1}$.

Nas Figuras 3a e 3b, perfis da concentração de nitrato em função do tempo são mostrados para reatores anóxicos em sistemas de fluxo contínuo. No reator pré-D (antes da aeração), a taxa de desnitrificação inicialmente é alta de acordo com a Eq. (8a), mas quando o material facilmente biodegradável é metabolizado a taxa se reduz e Eq. (8b) passa a descrever o processo. No reator pós $D$ (depois da aeração), a taxa é de acordo com a Eq (8c).

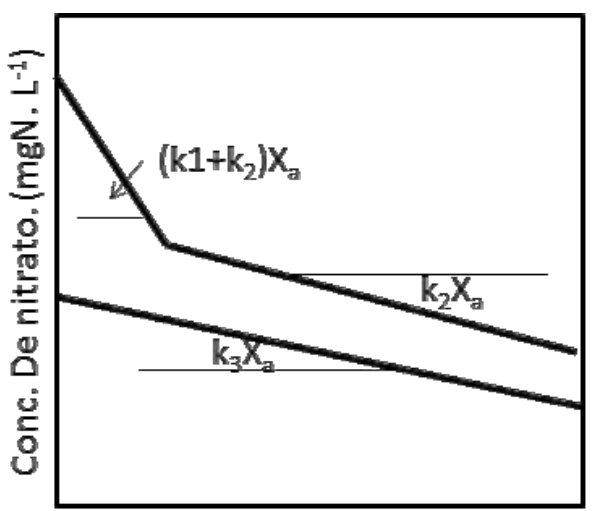

Figura 3a: Perfil típico de nitrato-tempo em reator anóxico de fluxo pistão.

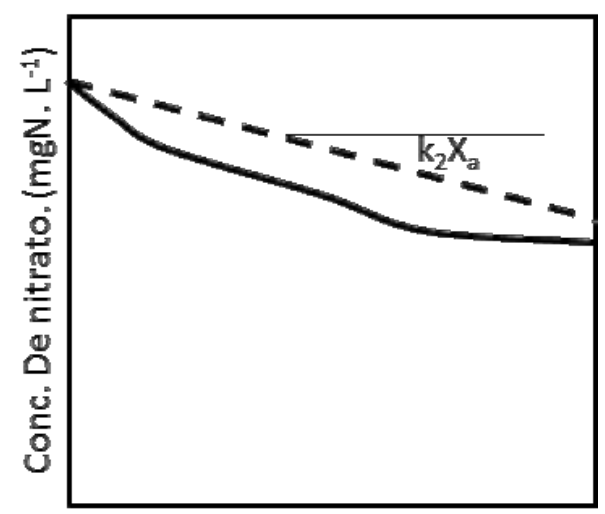

Figura 3b: Perfil nitrato-tempo em sistemas anóxicos RBS.

Em sistemas RBS, é inevitável que parte do material facilmente biodegradável seja usado por oxigênio dissolvido e, portanto, não esteja disponível para a desnitrificação. Em sistemas de RBS do tipo N-D, a taxa de desnitrificação tenderá a mudar à medida em que a batelada está sendo tratada. Isso é exemplificado na Figura 3b (curva desenhada). No ambiente anóxico, a taxa de desnitrificação é alta logo depois da alimentação de uma batelada, mas após o metabolismo da DQQ facilmente biodegradável a taxa diminui e pode ser descrita pela equação (8b). Se o tempo para o tratamento de uma batelada for suficientemente longo, eventualmente todo o material orgânico vai ser utilizado e a taxa será reduzida para o valor da Equação (8c). Em um sistema de N-D, onde a nitrificação é aplicada sem nitrato inicialmente presente, a taxa de desnitrificação experimental se aproxima da taxa teórica da Eq. (8b) ainda mais perto do que na Figura 2, por duas razões: (1) inicialmente, o nitrato não está disponível e o material facilmente biodegradável é metabolizado em alta taxa no reator aeróbio e (2), em geral, o tempo sem o material particulado vai ser curto ou mesmo inexistente. Portanto, a Eq. (8b) dá uma descrição adequada da taxa de desnitrificação no reator anóxico de um sistema de N-D.

\subsection{Modelação da remoção de nitrogênio em sistemas de fluxo contínuo}

As considerações básicas para a modelação do sistema Bardenpho são: (1) a produção de nitrato no reator aeróbio (que depende da concentração de amônio disponível no afluente, $\mathrm{N}_{\mathrm{c}}$ ) deve ser acompanhada por uma capacidade de remoção de nitrato nos reatores anóxicos, que depende da concentração de lodo ativo e da concentração da DQO biodegradável no afluente e (2) a recirculação de licor misto deve ser tal que o nitrato esteja realmente disponível para desnitrificação nos reatores anóxicos. VAN HAANDEL E MARAIS (1999) desenvolveram a seguinte equação:

$\left(N_{c} / S_{b a}\right)_{o}=(a+s+1)\left(f_{d n} f_{s b}+k_{2} C_{r} f_{m}\right) /\left[a+\left(k_{2} / k_{3}\right)(s+1)\right]$

Onde:

$\left(\mathrm{N}_{\mathrm{c}} / \mathrm{S}_{\mathrm{ba}}\right)_{\mathrm{o}}=$ razão máxima que permite remoção completa de nitrogênio; 
$\mathrm{N}_{\mathrm{c}}=$ concentração de nitrogênio no afluente que deve ser removida;

$\mathrm{S}_{\mathrm{ba}}=$ DQO biodegradável do afluente;

a, $s=$ taxas de recirculação da zona de nitrificação e do decantador (Fig. 1);

$\mathrm{f}_{\mathrm{dn}}=$ razão entre a remoção de nitrato e metabolismo da DQQO;

$=\left(1-f_{c v} Y\right) / 2,86=1 / 8,6=0,11 \mathrm{mgN} \cdot \mathrm{mg}^{-1} \mathrm{DQO}$;

$\mathrm{f}_{\mathrm{sb}}=$ fração da DQO biodegradável que é solúvel no afluente $(\approx 0,25)$;

$C_{r}=Y R_{s} /\left(1+b_{h} R_{s}\right)$

$\mathrm{f}_{\mathrm{m}}=$ fração máxima da massa de lodo em ambiente anóxico que permite nitrificação eficiente;

$=1-\left(1+K_{n} / N_{a d}\right)\left(b_{n}+1 / R_{s}\right) / \mu_{m}$ (VAN HAANDEL et al., 1981);

$\mathrm{N}_{\mathrm{ad}}=$ concentração desejada de amônia residual no efluente (por exemplo. $<2$ mg. $\mathrm{L}^{-1}$ );

$\mathrm{K}_{\mathrm{n}}=$ concentração de meia saturação de amônia para nitrificação.

A razão $\left(\mathrm{N}_{\mathrm{c}} / \mathrm{S}_{\mathrm{ba}}\right)_{0}$ representa a proporção entre o substrato da nitrificação $\left(N_{c}\right)$ e o da desnitrificação $\left(\mathrm{S}_{\text {ba }}\right)$. Essa razão pode ser transformada facilmente em razão NTK/DOQO do afluente que permite remoção completa de nitrogênio, $\left(\mathrm{N}_{\mathrm{ta}} / \mathrm{S}_{\mathrm{ta}}\right)_{0}$ :

$\left(\mathrm{N}_{\mathrm{ta}} / \mathrm{S}_{\mathrm{ta}}\right)_{\mathrm{o}}=\left(\mathrm{N}_{\mathrm{c}} / \mathrm{S}_{\mathrm{ba}}\right)\left(1-\mathrm{f}_{\mathrm{us}}-\mathrm{f}_{\mathrm{up}}\right)+\left(\mathrm{N}_{1}+\mathrm{N}_{\mathrm{te}}\right) / \mathrm{S}_{\mathrm{ta}}$

A idade de lodo mínima para haver desnitrificação se calcula para $\mathrm{f}_{\mathrm{m}}=0$, i.e. quando

$1-\left(1+K_{n} / N_{a d}\right)\left(b_{n}+1 / R_{s m}\right) / \mu_{m}=0$ ou

$R_{s m}=1 /\left(\mu_{m} /\left(1+K_{n} / N_{a d}\right)-b_{n}\right)$

Na Figura 4, a razão $\left(\mathrm{N}_{\mathrm{c}} / \mathrm{S}_{\mathrm{ba}}\right)_{0}$ e $\left(\mathrm{N}_{\mathrm{ta}} / \mathrm{S}_{\mathrm{ta}}\right)_{\mathrm{o}}$ estão plotados em função da idade do lodo. Para traçar esse gráfico, uma série de características do esgoto e de condições operacionais devem ser co- nhecidas. Estas são apresentadas na Tabela 1. As características das águas residuárias (coluna 1) são típicas para esgotos municipais, exceto os valores para a temperatura, que é um valor para as regiões tropicais. No que diz respeito às condições operacionais (coluna 2), esses valores são presumidos, compatíveis com valores comumente usados na prática. Os valores das constantes (coluna 3) dependem da temperatura e até certo ponto da origem da água residuária a ser tratada. A Fig. 4 mostra que nas condições de Tabela 1 e para uma proporção "normal” $\left(\mathrm{N}_{\mathrm{ta}} / \mathrm{S}_{\mathrm{ta}} \approx 0,1 \mathrm{mgTKN} \cdot \mathrm{mg}^{-1} \mathrm{D}\right.$ QO aproximadamente), a remoção completa de nitrogênio é possível quando a idade de lodo não é muito pequena. Na Fig. 4 , a idade de lodo de 8 $\mathrm{d}$ é ótima, porque possibilita $\mathrm{N}_{\mathrm{ta}} / \mathrm{S}_{\mathrm{ta}}=\left(\mathrm{N}_{\mathrm{ta}} / \mathrm{S}_{\mathrm{ta}}\right)_{0}=$ $0,10 \mathrm{mgN} \cdot \mathrm{mg}^{-1} \mathrm{DQ}$ O. Para idades de lodo maiores, a remoção completa de $\mathrm{N}$ é possível, mas o custo de investimento (maior volume) e de operação (maior consumo de energia) maiores é maior. Por essa razão não é recomendável usar uma idade de lodo maior do que oito dias para as condições da Tabela 1.

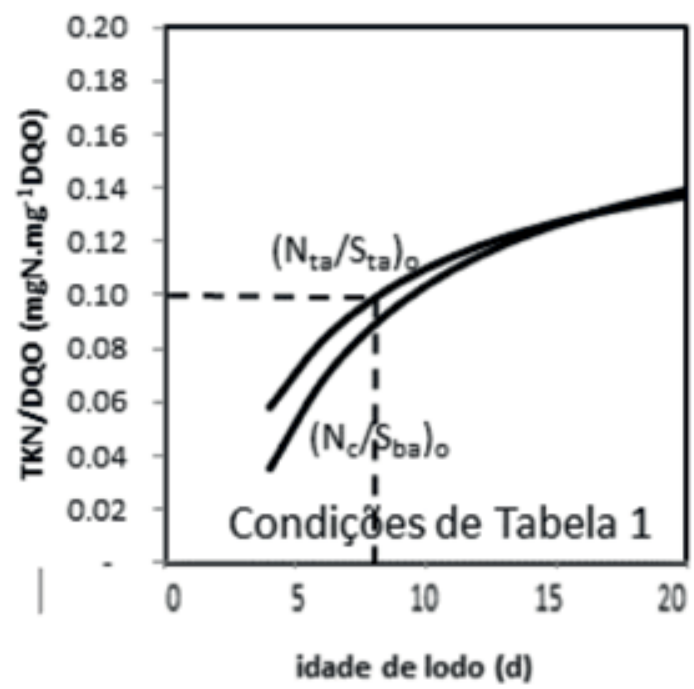

Figura 4: Razão máxima TKN/DQQO que pode ser removido num sistema Bardenpho como função da idade de lodo. A razão máxima (Nc/Sba)o também está indicada. 


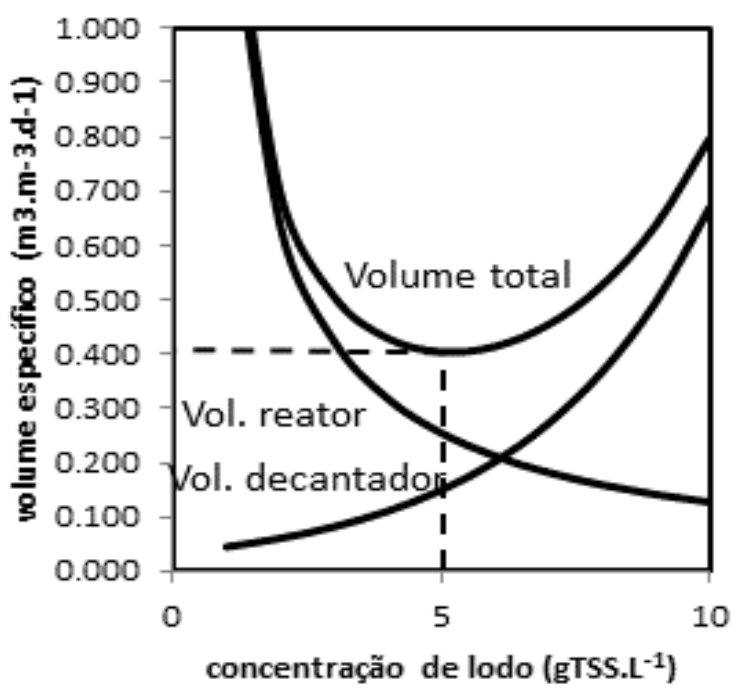

Figura 5: Volume específico de um sistema Bardenpho como função da concentração de lodo para uma idade de lodo ótima de Figura 4 e condições da Tabela 1.

Tabela 1: Características da água residuária, condições operacionais e constantes necessárias para calcular as taxas de nitrificação e desnitrificação em sistemas de lodo ativado.

\begin{tabular}{|c|c|c|c|c|c|}
\hline \multirow{2}{*}{\multicolumn{2}{|c|}{$\begin{array}{c}\text { Coluna } 1 \\
\begin{array}{l}\text { Características da água } \\
\text { residuária }\end{array}\end{array}$}} & \multirow{2}{*}{\multicolumn{2}{|c|}{$\begin{array}{c}\text { Coluna } 2 \\
\text { Condições } \\
\text { operacionais }\end{array}$}} & \multirow{2}{*}{\multicolumn{2}{|c|}{$\begin{array}{l}\text { Coluna } 3 \\
\text { Constantes } \\
\text { cinéticas }\end{array}$}} \\
\hline & & & & & \\
\hline $\mathrm{S}_{\mathrm{ta}}$ & $600 \mathrm{mg}^{-\mathrm{L}^{-1}}$ & $R_{h}$ & $0,5 \mathrm{~d}$ & $\mu_{\max }$ & $0,5 d^{-1}$ \\
\hline $\mathrm{N}_{\mathrm{ta}}$ & $60 \mathrm{mg} \cdot \mathrm{L}^{-1}$ & $R_{s}$ & $20 d$ & $\mathrm{k}_{2}$ & $\begin{array}{l}0,15 \mathrm{gN} / \\
\mathrm{gX}_{\mathrm{a}}-1 \mathrm{~d}^{-1}\end{array}$ \\
\hline $\mathrm{f}_{\text {us }}$ & 0,10 & $V_{b} / V_{r}$ & 0,5 & $\mathrm{k}_{3}$ & $\begin{array}{c}0,10 \mathrm{gN} / \\
\mathrm{gX}^{-1} \mathrm{~d}^{-1}\end{array}$ \\
\hline$f_{\text {up }}$ & 0,05 & $\mathrm{H}$ & $2 m$ & $k_{n}$ & $1 \mathrm{mg}^{-\mathrm{L}^{-1}}$ \\
\hline $\mathrm{f}_{\mathrm{bs}}$ & 0,2 & $\mathrm{a}$ & 4 & k & $0,3 \mathrm{~g} \cdot \mathrm{L}^{-1}$ \\
\hline$f_{v}$ & 0,75 & $\mathrm{~s}$ & 1 & $v_{0}$ & $10 \mathrm{~m} \cdot \mathrm{h}^{-1}$ \\
\hline Temperatura & $25^{\circ} \mathrm{C}$ & $\mathrm{N}_{\mathrm{ad}}$ & $2 \mathrm{ngN} \cdot \mathrm{L}^{-1}$ & $k_{0}$ & $1 \mathrm{mg}^{-\mathrm{L}^{-1}}$ \\
\hline
\end{tabular}

Em termos práticos, é de grande importância saber qual é o volume mínimo necessário para tratar águas residuárias com uma determinada composição. Isso só pode ser feito quando se conhecem os valores da concentração e composição do material orgânico afluente, bem como a sedimentabilidade. A sedimentabilidade de um lodo pode ser expressa pela equação proposta por Vesilind (1968), para determinação da velocidade de sedimentação:

$v=v_{0} \exp \left(-k X_{t}\right)$
Onde

$v$ = velocidade de sedimentação;

$v_{0}=$ constante que reflete a velocidade a velocidade de sedimentação não estorvada;

$\mathrm{k}=$ constante que reflete $\mathrm{a}$ compressibilidade do lodo.

Os valores das constantes $k$ e $v_{0}$ podem ser determinados experimentalmente se os valores das constantes de Vesilind são conhecidos (ou estimados), os valores ótimos de concentração para um valor mínimo do volume específico do sistema (volume de reatores + decantador por $\mathrm{m}^{3} \cdot \mathrm{d}^{-1} \mathrm{de}$ afluente) podem ser calculados, como mostram Van Haandel e Marais (1999).

$v_{r}=m X_{t} \cdot S_{t a} / X_{t}$

$v_{d}=s_{f} H /\left(v_{o} \exp \left(-k X_{t}\right)\right.$

Onde:

$v_{r}=$ volume específico dos reatores $\left(m^{3} \cdot m^{-3} \cdot d^{-1}\right)$;

$v_{d}=$ volume específico do decantador $\left(\mathrm{m}^{3} \cdot \mathrm{m}^{-3} \cdot \mathrm{d}^{-1}\right)$;

$\mathrm{s}_{\mathrm{f}}=$ fator de segurança $(\approx 2)$;

$\mathrm{H}=$ altura do decantador $(\approx 4 \mathrm{~m})$.

Como exemplo se vê na Figura 5 o volume dos reatores e do decantador, bem como o total em função da concentração de lodo para os dados de Tabela $1\left(R_{s}=8 d\right)$. A concentração ótima é $X_{t}$ $=5 \mathrm{~g} \cdot \mathrm{L}^{-1}$, porque para essa concentração o volume do sistema é mínimo: 149 e $255 \mathrm{~L} \cdot \mathrm{m}^{-3} \cdot \mathrm{d}^{-1}$ para o decantador e os reatores respectivamente e 404 L.m $\mathrm{m}^{-3} \cdot \mathrm{d}^{-1}$ para o sistema total. Esses cálculos agora podem ser feitos para qualquer idade de lodo e, assim, o projeto otimizado do sistema Bardenpho para remoção de nitrogênio pode ser definido em função dessa variável. Isso se mostra na Figura 6 , onde se evidencia que, mesmo para as boas propriedades de sedimentação do lodo que se supôs $\left(k=0,3 \mathrm{~L} \cdot \mathrm{g}^{-1}, \mathrm{v}_{0}=10 \mathrm{~m} \cdot \mathrm{h}^{-1}\right)$, o volume do decanta- 
dor é uma fração considerável do volume dos reatores para qualquer idade do lodo.

Uma vez que o volume específico do sistema tenha sido estabelecido, é fácil calcular a capacidade de remoção de nitrogênio, que é a quantidade de nitrogênio que pode ser removida por unidade de volume do sistema e por unidade de tempo. A capacidade de remoção de nitrogênio é a razão entre a concentração de nitrogênio que pode ser removida $\left(\mathrm{N}_{\mathrm{c}}\right)$ e o volume específico total. A Figura 7 mostra a capacidade de remoção de nitrogênio de um sistema Bardenpho em função da idade do lodo. No exemplo, pode claramente ser visto que a capacidade de remoção de nitrogênio passa por

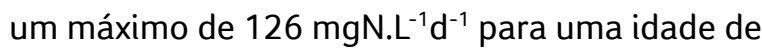
lodo de 10,4 d. A variável idade de lodo é importante para os custos de investimento porque define o volume mínimo do sistema (Figura 6), mas também para os custos operacionais porque influi no consumo de oxigênio e na produção de lodo. Se a remoção completa de nitrogênio não é possível num sistema Bardenpho, o nitrogênio residual estará no efluente principalmente como nitrato.

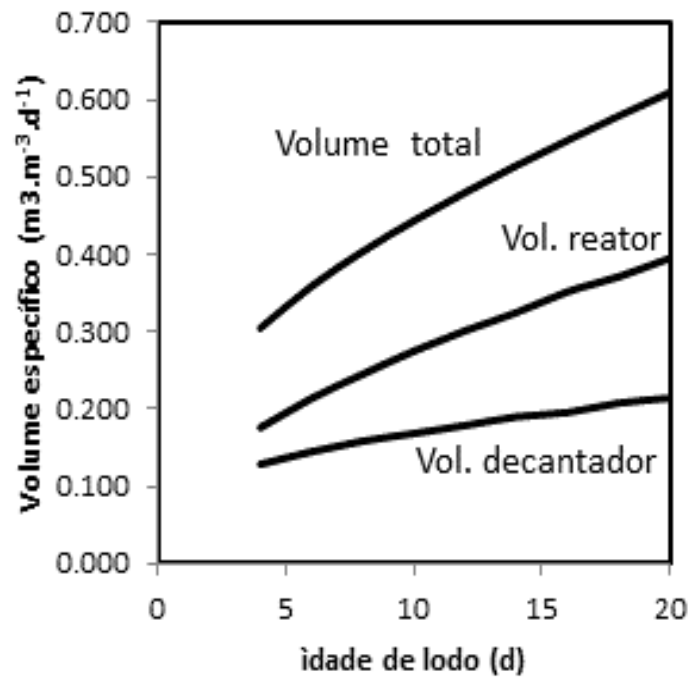

Figura 6: Volume específico de um sistema Bardenpho em função da idade de lodo para concentração ótima de lodo (Fig.5) e características de esgoto e condições operacionais da Tabela 1.

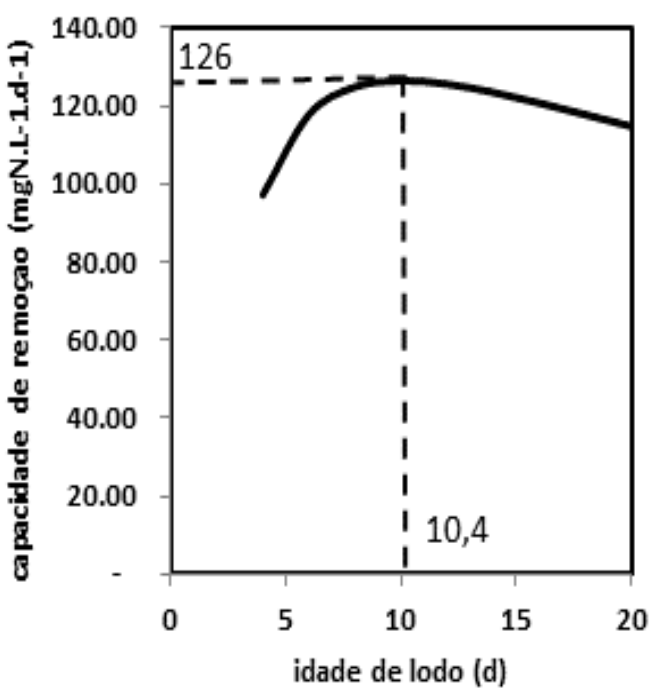

Figura 7: Capacidade máxima de remoção de nitrogênio de um sistema Bardenpho, num RBS N-D e RBS NDS com lodo granulado como função da idade de lodo para as especificações de Tabela 1.

\subsection{Modelação da remoção de nitrogênio no sistema RBS do tipo N-D}

No reator aeróbio do sistema N-D pode-se supor que a nitrificação ocorra a taxa máxima, já que nem amônia nem OD são limitantes, enquanto a batelada está sendo processada. No reator anóxico a taxa de desnitrificação pode ser expressa pela Eq. (8b). No sistema N-D, a capacidade de produção de nitrato no reator aeróbio será igual à capacidade de remoção de nitrato do reator anóxico se:

$C_{N}=V_{a e} r_{N}=C_{D}=V_{a n} r_{D}$

Portanto

$V_{a e}\left(\mu_{\max } / Y_{n}\right) X_{n}=V_{a n} k_{2} X_{a}$

ou

$V_{a e} \mu_{\max } /\left(1+b_{n} R_{s}\right) N_{c}=V_{a n} k_{2} Y /\left(1+b_{h} R_{s}\right) S_{b a}$

ou ainda

$\mathrm{r}_{\mathrm{N}} / \mathrm{r}_{\mathrm{D}}=\mathrm{V}_{\mathrm{an}} / \mathrm{V}_{\mathrm{ae}}=\left[\mu_{\max } /\left(1+\mathrm{b}_{\mathrm{n}} \mathrm{R}_{\mathrm{s}}\right)\right] /\left[\mathrm{k}_{2} \mathrm{Y} /\left(1+\mathrm{b}_{\mathrm{h}} \mathrm{R}_{\mathrm{s}}\right)\right]^{*}\left(\mathrm{~N}_{\mathrm{c}} /\right.$ $\mathrm{S}_{\mathrm{ba}}$ ) 
onde:

$\mathrm{C}_{\mathrm{N}}=$ capacidade de nitrificação no reator aeróbio;

$C_{D}=$ capacidade de desnitrificação no reator anóxico;

$\mathrm{V}_{\mathrm{ae}}=$ volume do reator aeróbio;

$\mathrm{V}_{\mathrm{an}}=$ volume do reator anóxico;

$\mathrm{S}_{\mathrm{ba}}=$ concentração da DQO biodegradável no afluente.

A partir da Eq. (15 d), pode-se notar que a proporção entre o volume do reator anóxico e do aeróbio depende das constantes cinéticas para a nitrificação e desnitrificação $\left(\mu_{\max }, b_{n}, k_{2}, b_{h}\right.$, que dependem todas da temperatura), a idade de lodo é a razão entre as concentrações do substrato a ser nitrificado $\left(\mathrm{N}_{\mathrm{c}}\right)$ e do substrato utilizado para desnitrificar $\left(\mathrm{S}_{\mathrm{ba}}\right)$. Assim, para qualquer proporção de $N_{c} / S_{b a}$ a razão entre o volume $V_{a e} / V_{a n}$ pode ser calculada como uma função da idade do lodo. Em princípio, a proporção indica a divisão do volume para a remoção completa de nitrogênio, mas existe uma condição limitante: a idade de lodo deve ser suficiente para a nitrificação ocorrer. Essa idade de lodo mínima do sistema N-D pode ser calculada como (VAN HAANDEL et al., 1981):

$\mathrm{R}_{\mathrm{sm}}=1 /\left[\mu_{\mathrm{m}} \mathrm{V}_{\mathrm{ae}} /\left(\mathrm{V}_{\mathrm{ae}}+\mathrm{V}_{\mathrm{an})}-\mathrm{b}_{\mathrm{n}}\right]\right.$

Para calcular a capacidade de remoção de nitrogênio do sistema RBS N-D, primeiramente deve ser estabelecido quanto tempo está disponível para o tratamento biológico. 0 tempo necessário para o tratamento de uma batelada de água residuária é composto por quatro partes: (1) descarga de bateladas de afluente no reator; (2) tratamento biológico; (3) sedimentação do lodo após o tratamento e (4) descarga do efluente tratado. Os passos (1) e (4) dependem de equipamento de bombeamento e podem ser considerados essencialmente constantes e curtos. Passo (3) depende da concentração total de lodo, $X_{\mathrm{t}}$, expressa na Equação (6).
O tempo de sedimentação pode ser calculado dividindo a altura que o lodo tem de sedimentar (a altura das bateladas de água residuária) pela velocidade de sedimentação:

$\mathrm{t}_{\mathrm{s}}=\mathrm{H} / \mathrm{v}=\mathrm{H} /\left(\mathrm{v}_{\mathrm{o}} \exp \left(-k X_{\mathrm{t}}\right)\right)$

Onde:

$\mathrm{H}=$ altura de sedimentação de lodo $=\mathrm{V}_{\mathrm{b}} / \mathrm{V}_{\mathrm{r}}{ }^{*} \mathrm{H}_{\mathrm{r}}$;

$\mathrm{H}_{\mathrm{r}}=$ altura do reator;

v = velocidade de sedimentação do lodo;

$\mathrm{k}, \mathrm{v}_{0}=$ Constantes de sedimentabilidade de Vesilind.

Em sistemas de lodo ativado com remoção de nitrogênio, normalmente o tempo necessário para a nitrificação é o fator determinante do tempo de aeração, e pode ser expresso como a razão entre a massa de nitrogênio a ser removido e a massa nitrificada ou desnitrificada por unidade de tempo:

$t_{b}=\left(N_{c} V_{b}\right) /\left(r_{N} V_{a e}\right)=\left(N_{c} V_{b}\right) /\left(r_{D} V_{a n}\right)$

Onde:

$\mathrm{t}_{\mathrm{b}}=$ tempo necessário para o tratamento biológico;

$\mathrm{N}_{\mathrm{c}}=$ concentração de amônia que deve ser removida no sistema;

$\mathrm{V}_{\mathrm{b}}=$ volume da batelada .

Conforme a Eq. (15), a proporção entre volumes aeróbio e anóxico depende das taxas de nitrificação e desnitrificação. Por exemplo, para as especificações da Tabela 1, calcula-se que para uma idade de lodo de 20 d as taxas de nitrificação e desnitrificação são:

$r_{N}=\left(\mu_{\max } / Y_{n}\right) X_{n}=0,5 / 0,1 * 0,1 * 20 /(1+0,05 * 20) 49 / 0,5=$ $490 \mathrm{mgN} \cdot \mathrm{L}^{-1} \cdot \mathrm{d}^{-1}=20,4 \mathrm{mg} \cdot \mathrm{L}^{-1} \cdot \mathrm{h}^{-1}$

$r_{D}=k_{2} x_{a}=0,15 * 0,45 * 20 /(1+0,3 * 20) 510 / 0,5=196$ $\mathrm{mg} \cdot \mathrm{L}^{-1} \mathrm{~d}^{-1}=8,2 \mathrm{mg} \cdot \mathrm{L}^{-1} \mathrm{~h}^{-1}$

Portanto: $V_{a n} / V_{a e}=r_{N} / r_{D}=20,4 / 8,2=2,49$

e 
$\mathrm{V}_{\mathrm{ae}} / \mathrm{V}_{\mathrm{r}}=\mathrm{V}_{\mathrm{ae}} /\left(\mathrm{V}_{\mathrm{ae}}+2,49 \mathrm{~V}_{\mathrm{ae}}\right)=0,29 \mathrm{eV}_{\mathrm{an}} / \mathrm{V}_{\mathrm{r}}=0,71$

Para otimizar o sistema N-D se aplica o seguinte procedimento, exemplificado na Tabela 2.

(1) Seleciona-se uma faixa de vazões do afluente. No exemplo da Tabela 2 escolheu-se um volume de $15 \mathrm{~L}$ e uma faixa de vazões de 30 a $50{\mathrm{~L} . \mathrm{d}^{-1}}^{-1}$ (coluna 1);

(2) Definidos os dados da Tabela 1, para determinada idade de lodo (no exemplo $R_{s}=20 \mathrm{~d}$ ) calcula-se os fatores que são determinados pela idade de lodo: a concentração de nitrogênio necessário para produzir lodo, $\mathrm{N}_{1}$, a concentração de nitrogênio a ser removida $N_{c}=N_{t a}-N_{1}-N_{t e}$ e os volumes aeróbio e anóxico. No exemplo usou-se um volume de $15 \mathrm{~L}$, para uma idade de lodo de $20 \mathrm{~d}$, portanto, calcula-se:

$\mathrm{N}_{1}=9,2 \mathrm{mg} \cdot \mathrm{L}^{-1}$ e Nc$=60-9,2-2=48.8 \mathrm{mg} \cdot \cdot^{-1}$

$V_{a n} / V_{a e}=\left[\mu_{\max } /\left(1+b_{n} R_{s}\right)\right] /\left[k_{2} Y /\left(1+b_{h} R_{s}\right)\right] *\left(N_{c} / S_{b a}\right)=$ 2.5, i.e. $V_{a e}=4,3$ e $V_{a n}=10,7 \mathrm{~L}$

(3) Para a faixa de vazões selecionada calculou-se as concentrações de $X_{n}$ e $X_{a}$ (colunas 2 e 3 ) e as taxas de nitrificação e desnitrificação (colunas 5 e 6);
(4) Com Eqs. 17 e 16 calculou-se os valores do tempo de aeração (coluna 7) e de sedimentação (coluna 8), bem como o tempo total de tratamento de uma batelada (coluna 9) para a faixa de vazões selecionadas;

(5) Calcule o número de bateladas por dia $\left(\mathrm{N}_{\mathrm{b}}=24 /\right.$ $t_{\mathrm{t}}$, coluna 10) e a vazão que pode ser tratada (coluna 11) como o produto $\mathrm{N}_{\mathrm{b}} \mathrm{V}_{\mathrm{b}}$;

(6) Agora se compara a vazão aplicada (coluna 1) com a vazão que pode ser tratada (coluna 11). Pode se observar no exemplo de Tabela 2 que para

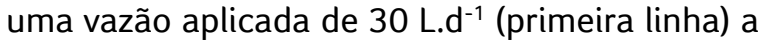
vazão que pode ser tratada é 35,6 L.d ${ }^{-1}$. Portanto, para essa vazão o sistema está subcarregado. Por outro lado, para uma vazão aplicada de 50 L.d ${ }^{1}$ (penúltima linha) a vazão que pode ser tratada é 40,3 L.d $^{-1}$. Portanto, para essa vazão o sistema está sobrecarregado;

(7) Por tentativas descobre-se que a vazão máxima que pode ser tratada é 40,9 L.d $\mathrm{d}^{-1}$ (última linha), quando a vazão aplicada é igual à vazão que pode ser tratada e o sistema funciona em capacidade máxima para a idade de lodo de $20 \mathrm{~d}$. Naturalmente com um computador pode-se estabelecer a vazão máxima com o "Solver" do Excel.

Tabela 2: Procedimento de identificação da vazão máxima que pode ser tratada sob as condições apresentadas na Tabela 1 idade de lodo de $20 \mathrm{~d}$.

\begin{tabular}{|c|c|c|c|c|c|c|c|c|c|c|}
\hline $\mathbf{1}$ & $\mathbf{2}$ & $\mathbf{3}$ & $\mathbf{4}$ & $\mathbf{5}$ & $\mathbf{6}$ & $\mathbf{7}$ & $\mathbf{8}$ & $\mathbf{9}$ & $\mathbf{1 0}$ & $\mathbf{1 1}$ \\
\hline $\mathbf{Q}$ & $\mathbf{X}_{\mathbf{n}}$ & $\mathbf{X}_{\mathbf{a}}$ & $\mathbf{X}_{\mathbf{t}}$ & $\mathbf{r}_{\mathbf{N}}$ & $\mathbf{r}_{\mathbf{D}}$ & $\mathbf{t}_{\mathbf{b}}$ & $\mathbf{t}_{\mathbf{s}}$ & $\mathbf{t}_{\mathbf{t}}$ & $\mathbf{N}_{\mathbf{b}}$ & $\mathbf{Q}$ \\
\hline 30 & 98 & 1,30 & 4,90 & 20,3 & 8,1 & 4,2 & 0,7 & 5,1 & 4,7 & 35,6 \\
\hline 34 & 111 & 1,47 & 5,55 & 23,1 & 9,2 & 3,7 & 0,8 & 4,7 & 5,1 & 38,2 \\
\hline 38 & 124 & 1,64 & 6,21 & 25,8 & 10,3 & 3,3 & 1,0 & 4,5 & 5,3 & 40,1 \\
\hline 42 & 137 & 1,82 & 6,86 & 28,5 & 11,4 & 3,0 & 1,2 & 4,4 & 5,5 & 41,1 \\
\hline 46 & 150 & 1,99 & 7,51 & 31,2 & 12,4 & 2,7 & 1,4 & 4,4 & 5,5 & 41,2 \\
\hline 50 & 163 & 2,16 & 8,16 & 33,9 & 13,5 & 2,5 & 1,7 & 4,5 & 5,4 & 40,3 \\
\hline $\mathbf{4 0 . 9}$ & $\mathbf{1 3 3}$ & $\mathbf{1 , 7 7}$ & $\mathbf{6 , 6 8}$ & $\mathbf{2 7 , 7}$ & $\mathbf{1 1 , 1}$ & $\mathbf{3 , 1}$ & $\mathbf{1 , 1}$ & $\mathbf{4 , 4}$ & $\mathbf{5 , 5}$ & $\mathbf{4 0 , 9}$ \\
\hline
\end{tabular}


Os cálculos na Tabela 2 indicam que o sistema tem uma capacidade de remoção de nitrogênio de $\mathrm{QaNc}=40,9 * 48,8=1996 \mathrm{mgN} . \mathrm{d}-1$ ou 1996/15 $=133 \mathrm{mgN} \cdot \mathrm{L}-1 \mathrm{~d}-1$ para a idade de lodo de $20 \mathrm{~d}$. Esse procedimento pode agora ser repetido para diferentes idades de lodo, estabelecendo-se assim a maior capacidade de remoção de nitrogênio do sistema. Na Figura 8 podem-se observar os valores do tempo de sedimentação e de aeração e ainda o tempo de processamento de uma batelada em função da idade de lodo. Na Figura 9 se apresenta a capacidade de remoção de nitrogênio em função da idade de lodo. A capacidade máxima encontrada é $138 \mathrm{mgN}$.L-1d-1 para a idade de lodo ótima do sistema, que é $13 \mathrm{~d}$. Conclui-se que esse valor é marginalmente maior que a capacidade do sistema Bardenpho, que era calculada em 126 mgN.L-1d-1 para uma idade de lodo de 10,4 d. Entretanto, nos cálculos se supôs que a sedimentabilidade do lodo no sistema Bardenpho e no sistema $\mathrm{N}-\mathrm{D}$ fossem iguais: $\mathrm{k}=0,3 \mathrm{~L} . \mathrm{g}-1$ e v0 $=10 \mathrm{~m} . \mathrm{h}-1$. Na realidade, o lodo do sistema em bateladas terá uma sedimentabilidade bem melhor, que leva a uma capacidade de remoção de nitrogênio bem maior. Por exemplo, quando se adota $\mathrm{k}=0,2 \mathrm{~L} . \mathrm{g}-1$, um valor perfeitamente realista para um sistema RBS, a capacidade de remoção de nitrogênio aumenta para $174 \mathrm{mg} . \mathrm{L}-1 \mathrm{~d}-1$ para uma idade de lodo de $16 \mathrm{~d}$ como mostra também a Figura 9. Portanto, para a mesma capacidade de remoção de nitrogênio, o volume do sistema RBS $\mathrm{N}$-D pode ser até $1-126 / 174=28 \%$ menor que 0 volume do sistema Bardenpho.

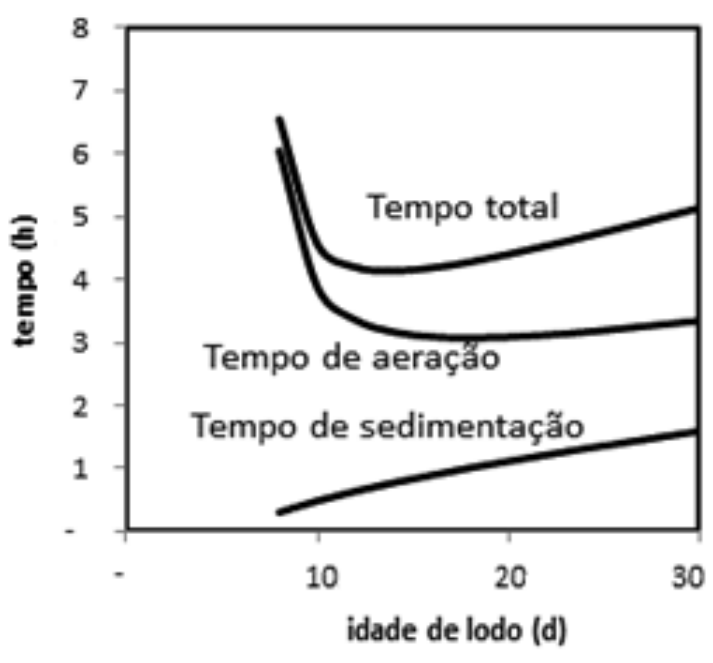

Figura 8: Tempo de sedimentação e do tratamento biológico em sistemas RBS em função de Rs $(k=0,4 \mathrm{l} / \mathrm{g}$; vo $=10 \mathrm{~m} / \mathrm{h}$ ).

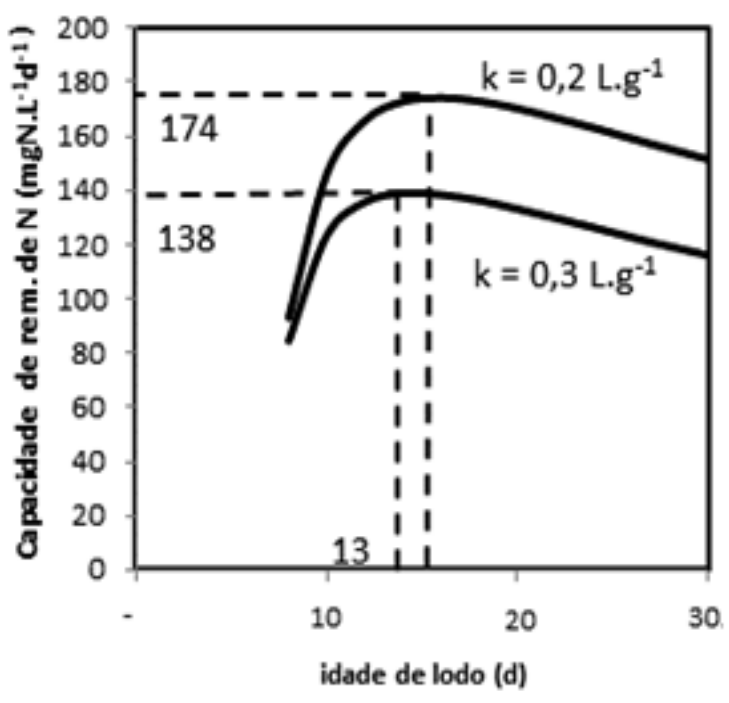

Figura 9: Número de bateladas por dia que podem ser tratadas em um sistema N-D sob as condições da Tabela 1. 


\subsection{Modelo para o sistema NDS usando lodo granulado}

Em sistemas RSB com nitrificação e desnitrificação simultâneas (NDS), as taxas de $r_{N}$ e $r_{D}$ de novo devem ser iguais, de modo que o nitrato é removido à medida em que é produzido. As mesmas expressões básicas para as taxas de nitrificação e desnitrificação, usadas para modelar o sistema $\mathrm{N}-\mathrm{D}$, podem novamente ser aplicadas, mas agora ambas têm valores reduzidos, devido à presença de uma concentração limitada de oxigénio dissolvido (OD). A influência da concentração de OD sobre a taxa de nitrificação e desnitrificação pode ser expressa como

$r_{N}=r_{N \max } O D /\left(O D+k_{0}\right)$

e

$r_{D}=k_{2}^{\prime} X_{a}$

Onde:

$r_{\mathrm{N} \max }=\left(\mu_{\max } / Y\right) X_{n} ;$

$\mathrm{k}_{2}{ }^{\prime}=$ constante de desnitrificação com valor reduzido devido à limitação de OD.

Para encontrar o valor da concentração de $O D$ para o qual $r_{N}=r_{D}$, uma investigação experimental foi realizada com lodo granular. Para essa finalidade, uma amostra de lodo granular foi obtida de um sistema operando em grande escala na Holanda e descrito por Pronk et al (2015). Essa amostra de lodo foi usada como inóculo para o RSB - sistema NDS em escala piloto ( $15 \mathrm{~L}$ ) operado a uma temperatura de $25^{\circ} \mathrm{C}$ e a uma idade do lodo de 20 dias, enquanto era alimentada com esgoto da cidade de Campina Grande (PB) por um período de seis semanas. Testes foram realizados para avaliar se o sistema NDS poderia ser operado uma eleva- da concentração de OD e ainda assim apresentar uma elevada taxa de desnitrificação. A investigação experimental foi composta de duas partes: (1) uma investigação específica para determinar a constante de meia saturação para OD de nitrificação do lodo granular e (2) uma investigação para determinar as taxas de nitrificação e desnitrificação como uma função da concentração de OD.

\section{(1) Constantes de nitrificação para lodo granular}

O valor da constante de meia saturação de OD foi determinado usando respirometria como uma ferramenta. Cloreto de amônio foi adicionado ao lodo, e a taxa de consumo de consumo de oxigênio (TCO) foi determinada para diferentes faixas de concentração de OD. A Figura 10 mostra um respirograma típico. Esse respirograma foi obtido com um respirômetro automatizado acoplado a um computador, que funciona sob as seguintes condições: uma batelada era aerada até atingir uma concentração de OD máxima prefixada. Em seguida, a aeração era interrompida pelo software do respirômetro, enquanto a agitação da amostra continuava para manter uma suspensão uniforme. Durante o período sem aeração, a concentração de OD diminuía devido ao consumo, até que um limite inferior, também prefixado, fosse alcançado e a aeração era retomada. $O$ software calculou a taxa de consumo de oxigénio (TCO) a partir da inclinação da reta OD-tempo durante os períodos sem aeração. Tanto a concentração de OD e TCO eram representadas graficamente em função do tempo no monitor do computador, enquanto o ensaio prosseguia. O respirograma assim obtido é a chave para os cálculos da taxa de crescimento máxima, $\mu_{\max }$ e a constante de meia saturação, $\mathrm{k}_{\mathrm{o}}$. O procedimento de cálculo é o seguinte: 


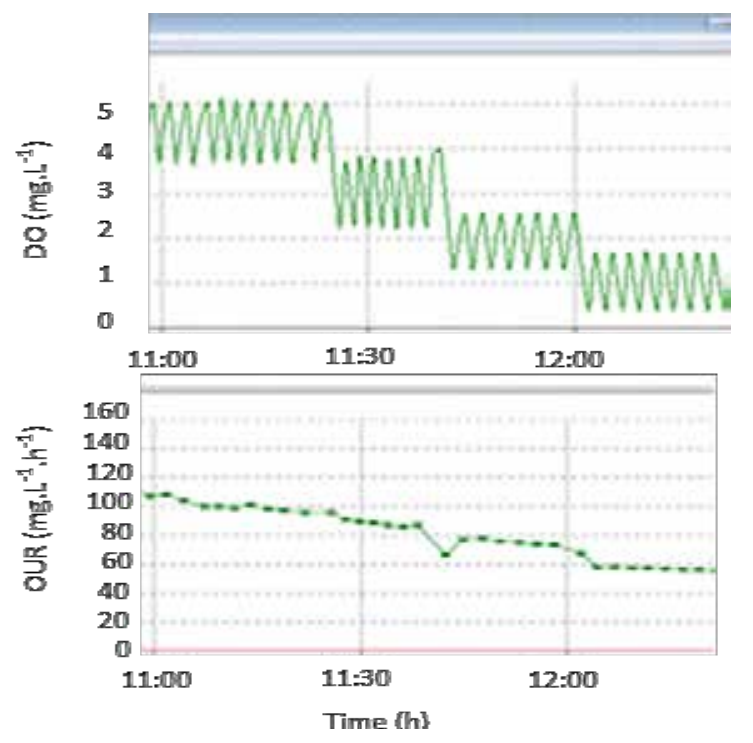

Figura 10: Respirograma mostrando a TCO (gráfico inferior) em função do tempo quando se aplicam diferentes concentrações de OD.

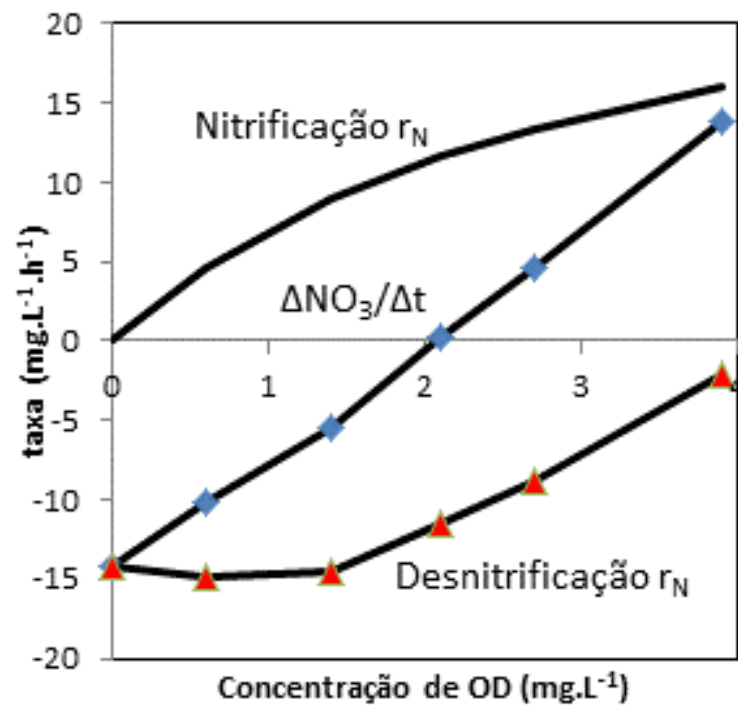

Figura 11: Taxa de nitrificação e desnitrificação e taxa de variação da concentração de nitrato em função da concentração de OD.

Tabela 3: Dados respirométricos de Fig. 10 para determinar as constantes de nitrificação de uma batelada de lodo granulado: Taxa máxima de crescimento de nitrificantes e constante de meia saturação de OD.

\begin{tabular}{|c|c|c|c|c|c|c|c|c|c|}
\hline 1 & 2 & 3 & 4 & 5 & 6 & 7 & 8 & 9 & 10 \\
\hline $\begin{array}{l}\text { Faixa de } \\
\text { OD }\end{array}$ & OD médio & TCO (tot) & TCO (nit) & rN (exp.) & $\mathrm{ko}=2,5$ & $\mathrm{ko}=2.75$ & $\mathrm{ko}=3,0$ & $\mathrm{ko}=3.25$ & ko $=3,06$ \\
\hline \multicolumn{10}{|c|}{$\mathbf{R}_{\mathrm{N} \max }$} \\
\hline $4-4.4$ & 4.2 & 100 & 75 & 16.4 & 26.2 & 27.1 & 28.1 & 29.1 & 28.3 \\
\hline $2.4-3.4$ & 2.8 & 92 & 67 & 14.7 & 27.8 & 29.1 & 30.5 & 31.8 & 30.8 \\
\hline $1.5-2.5$ & 2.2 & 78 & 53 & 11.6 & 24.8 & 26.1 & 27.4 & 28.7 & 27.7 \\
\hline $0.6-1.5$ & 1.2 & 49 & 36 & 7.9 & 24.7 & 26.3 & 28.0 & 29.7 & 28.4 \\
\hline \multicolumn{5}{|c|}{ Média de $r_{N \max }$} & 25.9 & 27.2 & 28.5 & 29.8 & 28.8 \\
\hline \multicolumn{5}{|c|}{ Desvio padrão } & 1.48 & 1.38 & 1.338 & 1.354 & 1.337 \\
\hline
\end{tabular}

(1) Usando a equação (1) calcular a concentração bactérias autotróficas e heterotróficas na amostra de lodo granulado. No caso particular de operação que gerou o lodo de Fig. 10, esses valores foram $X_{n}=132 \mathrm{mg}^{-1} \mathrm{~L}^{-1}$ (Equação (1)) e Xa $=1540$ $\mathrm{mg} \mathrm{L}^{-1}$ (Equação (2));

(2) Determinar a taxa de respiração endógena da amostra prolongando a aeração até a TCO ficar praticamente constante. No caso de o respirograma da Figura 10, foi observado um valor de $25 \mathrm{mg}$ $\mathrm{L}^{-1} \mathrm{~h}^{-1}$ (não mostrado no respirograma);
(3) Adicionou-se uma solução de cloreto de amônia à amostra assim preparada e observou-se a TCO em função do tempo, diminuindo-se gradualmente a concentração média de $O D$. $O$ respirograma mostra os valores de OD em função do tempo. Na tabela 3 podem-se observar as faixas de OD (coluna 1), bem como a concentração média de OD (coluna 2);

(4) As taxas de consumo de oxigênio para a nitrificação são determinadas subtraindo a taxa de respiração endógena do TCO total observado 
para cada uma das concentrações de OD (Tabela 3, coluna 3 e 4);

(5) As taxas de nitrificação agora são calculadas sabendo que há uma relação linear entre o consumo de oxigênio e a produção de nitrato. $O$ valor estequiométrico é $64 / 14=4,57 \mathrm{mgO} \cdot \mathrm{mg}^{-1} \mathrm{~N}$. As taxas de nitrificação, $\mathrm{r}_{\mathrm{N}}$, estão na coluna 5 ;

(6) Uma estimativa da taxa máxima de nitrificação, $r_{\text {Nmax }}$, e constante de meia saturação, ko, pode agora ser feita: $r_{N \max }=r_{N}\left(O D+k_{o}\right) / O D$. No entanto, o valor da meia saturação não é conhecido ainda. O problema é resolvido por um processo de tentativa e erro exemplificado na Tabela 3: para diferentes valores da constante $\mathrm{k}_{\mathrm{o}}$ e $\mathrm{r}_{\mathrm{N}}$ e as diferentes concentrações de OD os valores de $r_{\text {Nmax }}$ são calculados nas colunas 6 a 9 . 0 ideal para o verdadeiro valor de $\mathrm{k}_{\mathrm{o}} \mathrm{o}$ valor de $\mathrm{r}_{\mathrm{Nmax}}$ deve ser constante, independentemente da concentração de OD e igual à média e o desvio-padrão deve ser zero. Devido a erros experimentais, o valor de $r_{\mathrm{Nmax}}$ não será exatamente constante para qualquer valor $k_{o}$ e para todos os valores de um desvio padrão $k_{0}$ pode ser calculada. A melhor estimativa para $\mathrm{k}_{\mathrm{o}}$ é o valor que produz o menor desvio padrão. Por tentativa e erro, neste caso: $\mathrm{k}_{\mathrm{o}}=3,06 \mathrm{mg} \cdot \mathrm{L}^{-1}$ (coluna 10). A melhor estimativa para a taxa máxima de nitrificação é o valor médio para $\mathrm{k}_{\mathrm{o}}=3,06 \mathrm{mg} \cdot \mathrm{L}^{-1}: \mathrm{r}_{\mathrm{Nmax}}=$ $28,8 \mathrm{mgN} \cdot \mathrm{L}^{-1} \mathrm{~h}^{-1}$;

(7) Agora, a taxa de crescimento máximo das nitrificantes pode ser facilmente calculado: $r_{N \max }=\left(\mu_{\max }\right)$ $\left.Y_{n}\right) X_{n}$. Para as condições prevalecentes durante a geração do lodo granular, a concentração das nitrificantes foi calculada com a Equação (1): $X_{n}=132$ mg. $L^{-1}$. Assim:. $\mu_{\max }=28 \cdot 8 * 0 \cdot 1 * 24 / 1328=0,52 \mathrm{~d}^{-1}$.

\section{(2) Constantes de desnitrificação para lodo granular}

Para determinar a influência da concentração de OD sobre a taxa de desnitrificação, uma segunda investigação foi realizada com o mesmo lodo de- pois da remoção de amônia. Nessa investigação, bateladas de esgoto foram descarregadas no sistema RBS e a taxa de variação da concentração de nitrato foi observada como uma função do tempo para uma concentração de OD específico, que foi criada mantendo uma determinada capacidade de aeração. Depois de cada alimentação, um período de aeração de 30 minutos foi aplicado para remover o material facilmente biodegradável e nitrato foi adicionado ao afluente quando a concentração de OD foi baixa e o nitrato foi sendo consumido $\left(r_{D}>r_{N}\right)$. Em seguida, foram retiradas amostras em intervalos de 15 em 15 minutos, e a taxa de variação da concentração de nitrato foi determinada para cada uma das concentrações de OD. Os resultados estão na Tabela 4. Nas duas primeiras colunas, mostram-se a concentração de OD e a taxa observada de variação de nitrato devido à nitrificação e desnitrificação simultânea. $A$ coluna 3 mostra a taxa de nitrificação, calculada a partir da Equação (7b) para $\mu_{\max }=0,52 \mathrm{~d}^{-1}$ e $\mathrm{k}_{\mathrm{o}}=$ 3,06 mg. L-1 , obtidos no experimento com respirômetro, para as diferentes concentrações de OD. Finalmente se calculou a taxa de desnitrificação (coluna 4) como a diferença entre a taxa de variação da concentração de nitrato de (coluna 2) e a taxa de nitrificação (coluna 3). As diferentes taxas são apresentadas traçadas como uma função de OD na Figura 11.

A partir dos dados da Tabela 4 ou na Fig. 11 se estabelece que as taxas de nitrificação e desnitrificação são iguais quando a concentração de OD é de 2 $\mathrm{mg} . \mathrm{L}^{-1}$ e que, para essa concentração de OD, a taxa de nitrogênio emoção é igual $a r_{N}=r_{D}=11,5 \mathrm{mg}$.L${ }^{-1} \cdot h^{-1}$. Sabendo-se que a concentração de lodo ativo durante a investigação foi $1540 \mathrm{mg}^{\mathrm{L}} \mathrm{L}^{-1}$, a constante de desnitrificação é calculada com Eq (8) como $k_{2}{ }^{\prime}=0,18 \mathrm{mgN} \cdot \mathrm{mg}^{-1} X_{\mathrm{a}} \cdot \mathrm{d}^{-1}$. Esse valor é um pouco maior do que a constante proposta para lodo floculento à mesma temperatura $\left(25^{\circ} \mathrm{C}: 0,15 \mathrm{mgN}\right.$. $\mathrm{mg}^{-1} \mathrm{X}_{\mathrm{a}} \cdot \mathrm{d}^{-1}$ (VAN HAANDEL et al, 1981). Isso pode ser atribuído ao fato de que o lodo granular também removia fósforo em excesso, embora o siste- 
ma não fosse operado especificamente para essa finalidade. De todo modo, a remoção de $\mathrm{P}$ em excesso revela a presença de organismos acumuladores de fosfato (PAO em inglês), que são conhecidos por terem uma constante de desnitrificação bem maior que a das bactérias ordinárias (CLAYTON et al, 1991).

Tabela 4: Valores experimentais da taxa de variação da concentração de nitrato e valores correspondentes da taxa de nitrificação e de desnitrificação no reator NDS com lodo granular

\begin{tabular}{|c|c|c|c|}
\hline $\mathbf{1}$ & $\mathbf{2}$ & $\mathbf{3}$ & $\mathbf{4}$ \\
\hline Valor de OD & $\begin{array}{c}\Delta \mathrm{NO}_{3} / \mathbf{\Delta t} \\
\text { (exper.) }\end{array}$ & $\mathbf{r}_{\mathrm{N}}$ (intrap) & rD (calcul) \\
\hline 0 & -14.2 & 0 & -14.2 \\
\hline 0.6 & -10.8 & 4.7 & -14.9 \\
\hline 1.4 & -2.5 & 9.0 & -14.5 \\
\hline 2.1 & -0.2 & 11.6 & -11.4 \\
\hline 2.7 & 4.6 & 13.4 & -8.8 \\
\hline 3.9 & 13.9 & 16.1 & -2.2 \\
\hline
\end{tabular}

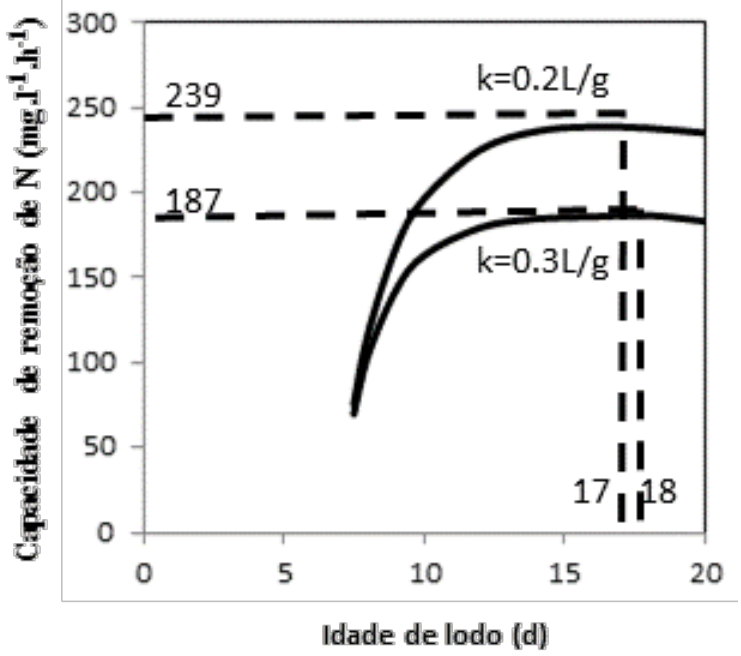

Figura 12: Capacidade de remoção de $\mathrm{N}$ do sistema NDS - RBS em função da idade de lodo para lodo granular com constante de compressibilidade $\mathrm{k}$ de 0,2

$$
\text { e } 0,3 \text { L.g }^{-1}
$$

Para estimar a taxa de desnitrificação para outras concentrações de OD, pode-se propor um modelo baseado na linearização desse parâmetro: usando os dados experimentais da Fig. 11, pode-se afir- mar que em boa aproximação a taxa de desnitrificação é dada por:

Para OD $<2$ mg. $\mathrm{L}^{-1}: \quad r_{\mathrm{D}}=\mathrm{r}_{\mathrm{Dmax}}$

Para $2<O D<4 \mathrm{mg} \cdot \mathrm{L}^{-1}: r_{D}=r_{\text {Dmax }}(2-0.50 D) \quad(21 \mathrm{~b})$

Para OD> $4 \mathrm{mg} \cdot \mathrm{L}^{-1}: \quad \mathrm{r}_{\mathrm{D}}=0$

\section{Otimização do sistema NDS com lodo granular}

A partir dos dados experimentais e do modelo, é evidente que uma concentração de OD de $2 \mathrm{mg} \cdot \mathrm{L}^{-1}$ é interessante. Valores inferiores a $2 \mathrm{mg} \mathrm{L}^{-1}$ reduzem a taxa de nitrificação, mas não aumentam substancialmente a taxa de desnitrificação. Por outro lado, valores superiores a $2 \mathrm{mg} \mathrm{L}^{-1}$ tendem a reduzir rapidamente a taxa de desnitrificação. Por isso, o valor de $\mathrm{OD}=2 \mathrm{mg} . \mathrm{L}^{-1}$ está perto do valor ótimo. Se for admitido que as Eqs (21a, b e c) também valem para outras idades do lodo, a taxa de desnitrificação pode ser calculada para qualquer idade de lodo e uma concentração de OD de $2 \mathrm{mg} \mathrm{L}^{-1}$, sabendo que, para qualquer idade de lodo, como pode ser visto na Eq (8b), é válido:

$r_{D}=r_{D \max }=k_{2}^{\prime} X_{a}$

Por outro lado, para qualquer concentração de OD a taxa de nitrificação é:

$r_{N}=r_{N \max } O D /\left(O D+k_{o}\right)=\left(\mu_{\max } / Y\right) X_{n}(2 / 5.1)=0.39\left(\mu_{m a-}\right.$ (Y) $X_{n}$

Para a concentração de OD de $2 \mathrm{mg} \mathrm{L}^{-1}$, a taxa de nitrificação tende a ser ligeiramente menor do que a taxa de desnitrificação. Assim, o tempo do período de aeração deve ser calculado a partir da taxa de nitrificação, uma vez que leva mais tempo do que a desnitrificação. A capacidade de remoção de nitrogênio no sistema agora pode ser calculada da mesma maneira, como exemplificado na Tabela 2 para a lodo floculento num sistema D-N. O tempo para o processamento biológico [Eq 
(18a)] agora tem de ser reformulado para a nova situação:

$\mathrm{t}_{\mathrm{b}}=\mathrm{N}_{\mathrm{c}}\left(\mathrm{V}_{\mathrm{b}} / \mathrm{V}_{\mathrm{r}}\right) / \mathrm{r}_{\mathrm{N}}$

A Tabela 5 mostra a vazão máxima que pode ser tratada em um sistema de NDS-RBS com lodo granular sob as condições da Tabela 1, com uma idade de lodo de $20 \mathrm{~d}$ e para uma constante de compressibilidade de lodo $\mathrm{k}=0.3 \mathrm{~L} \cdot \mathrm{g}^{-1}$. Tendo-se estabelecido a vazão máxima, os tempos para as fases no tratamento das bateladas, a capacidade de remoção de nitrogênio do sistema é agora facilmente calculada como: $\mathrm{N}_{\mathrm{c}} * \mathrm{~N}_{\mathrm{b}} * \mathrm{~V}_{\mathrm{b}} / \mathrm{V}_{\mathrm{r}}=52,8 * 6,9 * 0,5=183$
$\mathrm{mgN} \cdot \mathrm{L}^{-1} \cdot \mathrm{d}^{-1} \cdot \mathrm{O}$ mesmo processo de cálculo pode ser realizado por outras idades do lodo e a capacidade de remoção de nitrogênio pode ser calculada como uma função da idade do lodo. Na Figura 12, a relação é mostrada para $\mathrm{k}=0,3$ e $\mathrm{k}=0,2 \mathrm{~L} \cdot \mathrm{g}^{-1}$. Quando a capacidade de remoção de nitrogênio na Fig. 12 para lodo granular é comparada com a capacidade do sistema $\mathrm{N}-\mathrm{D}$ equivalente, mas com lodo floculento (Fig. 9), pode-se notar claramente que a capacidade de remoção de $\mathrm{N}$ do sistema NDS-RBS é superior ao sistema N-D porque, para as mesmas constantes de sedimentabilidade, o primeiro tem uma capacidade de $25 \%$ a $35 \%$ maior que o último.

Tabela 5: Determinação da vazão máxima que pode ser tratada num sistema NDS RBS de $15 \mathrm{~L}$ operado sob as condições de Tabela $1\left(\mathrm{~N}_{\mathrm{c}}=52.8 \mathrm{mg} \cdot \mathrm{L}^{-1}\right)$.

\begin{tabular}{|c|c|c|c|c|c|c|c|c|c|c|}
\hline $\mathbf{1}$ & $\mathbf{2}$ & $\mathbf{3}$ & $\mathbf{4}$ & $\mathbf{5}$ & $\mathbf{6}$ & $\mathbf{7}$ & $\mathbf{8}$ & $\mathbf{9}$ & $\mathbf{1 0}$ & $\mathbf{1 1}$ \\
\hline $\mathbf{Q}_{\mathbf{a}}$ & $\mathbf{X}_{\mathbf{n}}$ & $\mathbf{X}_{\mathbf{a}}$ & $\mathbf{X}_{\mathbf{t}}$ & $\mathbf{r}_{\mathbf{N}}$ & $\mathbf{r}_{\mathbf{D}}$ & $\mathbf{t}_{\mathbf{b}}$ & $\mathbf{t}_{\mathbf{s}}$ & $\mathbf{t}_{\mathbf{t}}$ & $\mathbf{N}_{\mathbf{b}}$ & $\mathbf{Q}$ \\
\hline 30 & 105.6 & 1311.4 & 3848.6 & 8.6 & 9.8 & 3.1 & 0.6 & 3.9 & 6.1 & 46.0 \\
\hline 34 & 119.6 & 1486.3 & 4361.8 & 9.7 & 11.1 & 2.7 & 0.7 & 3.7 & 6.6 & 49.2 \\
\hline 38 & 133.7 & 1661.1 & 4874.9 & 10.9 & 12.5 & 2.4 & 0.9 & 3.5 & 6.9 & 51.5 \\
\hline 42 & 147.8 & 1836.0 & 5388.1 & 12.0 & 13.8 & 2.2 & 1.0 & 3.4 & 7.0 & 52.9 \\
\hline 46 & 161.9 & 2010.9 & 5901.2 & 13.2 & 15.1 & 2.0 & 1.2 & 3.4 & 7.1 & 53.2 \\
\hline 50 & 175.9 & 2185.7 & 6414.4 & 14.3 & 16.4 & 1.8 & 1.4 & 3.4 & 7.0 & 52.7 \\
\hline 54 & 190.0 & 2360.6 & 6927.5 & 15.4 & 17.7 & 1.7 & 1.6 & 3.5 & 6.8 & 51.3 \\
\hline $\mathbf{5 2 . 1}$ & $\mathbf{1 8 3 . 3}$ & $\mathbf{2 2 7 6 . 6}$ & $\mathbf{6 6 8 1 . 2}$ & $\mathbf{1 4 . 9}$ & $\mathbf{1 7 . 1}$ & $\mathbf{1 . 8}$ & $\mathbf{1 . 5}$ & $\mathbf{3 . 5}$ & $\mathbf{6 . 9}$ & $\mathbf{5 2 . 1}$ \\
\hline
\end{tabular}

\subsection{Modelo para sistemas NDS usando lodo floculento}

O procedimento para otimizar a remoção de nitrogênio no sistema NDS descrito acima para lodo granular pode mutatis mutandis também ser utilizado para lodo floculento. VAN HAANDEL et al (2009) investigaram a influência da concentração de OD sobre nitrificação e desnitrificação em reatores NDS com lodo floculento. A Figura 13 resume os resultados experimentais. Pode-se notar que a influência da concentração de OD sobre as taxas de desnitrificação é muito mais forte do que no caso de lodo granular: a uma concentração de OD de $0,5 \mathrm{mg} \mathrm{L}^{-1}$, a desnitrificação é praticamente inexistente, e para forçar uma condição tal que $r_{N}=r_{D}$, a concentração de
OD é de cerca de $0,25 \mathrm{mg} \mathrm{L}^{-1}$. Na prática é muito difícil manter esse valor tão baixo, especialmente em reatores grandes. Mais importante ainda, sob essas condições, tanto a nitrificação como a desnitrificação taxas são muito baixas e, como resultado, a capacidade de remoção de nitrogênio é muito menor do que no caso de lodo granular. Isso é mostrado na Figura 14, onde a capacidade de remoção de nitrogênio é representada graficamente como uma função da idade de lodo para um sistema NDS optimizado com lodo floculento $\left(k=0,3 \mathrm{Lg}^{-1}\right)$ e cinética de nitrificação da Fig. 13 $\left(k_{2}=0,15 \mathrm{mgN} \cdot \mathrm{mg}^{-1} \mathrm{X}_{\mathrm{a}} \cdot \mathrm{d}^{-1}\right)$. A capacidade de remoção de nitrogênio com um máximo de cerca de $90 \mathrm{mg} \mathrm{L}^{-1} \cdot \mathrm{d}^{-1}$ é muito mais baixa do que nos três sistemas que foram discutidos antes. 


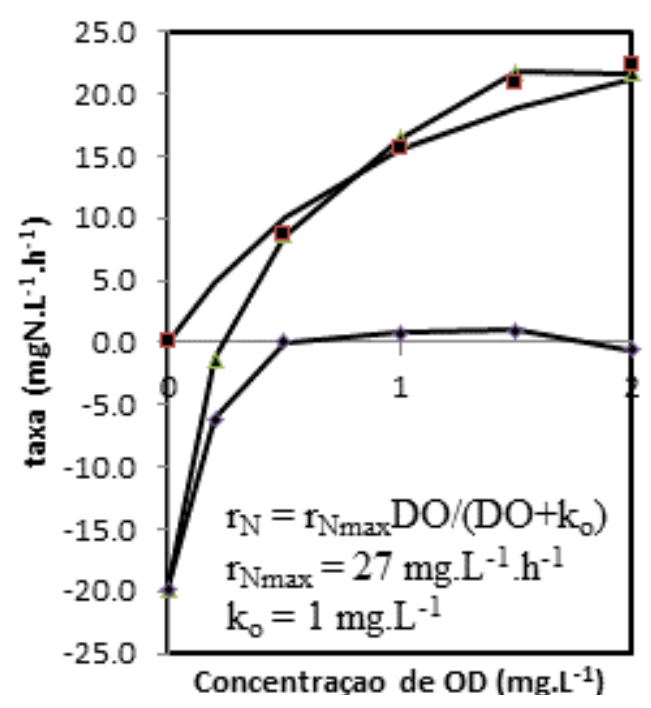

Figura 13: Taxa de variação de nitrato e taxas de nitrificação e desnitrificação em função da concentração de OD num sistema NDS com lodo floculento.

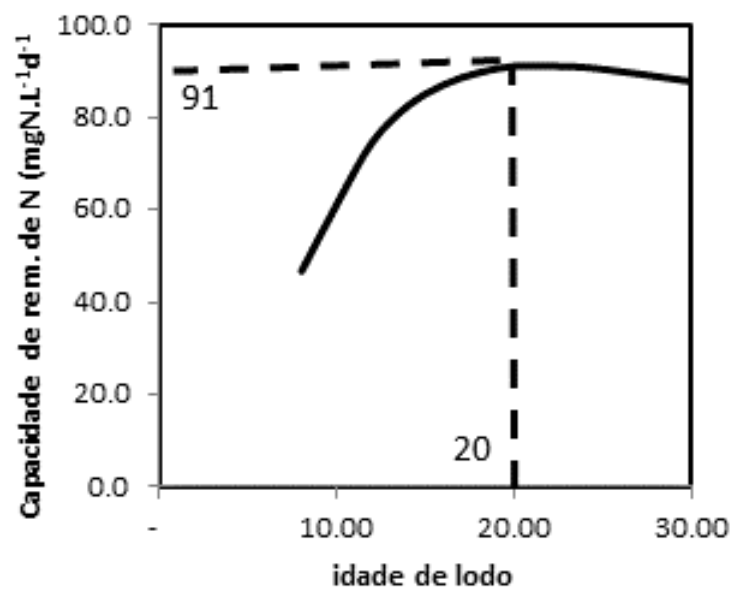

Figura 14: Capacidade de remoção de nitrogênio de um sistema NDS com lodo floculento em função da idade de lodo para as condições de Tabela 1.

\section{DISCUSSÃO}

Está amplamente comprovado que tanto sistemas de fluxo contínuo como as configurações Bardenpho ou UCT ou sistemas RBS de lodo ativado podem remover nitrogênio de forma eficiente de águas residuárias. Portanto, para comparar a eficiência das variantes do sistema de lodo ativado, a questão importante não é se elas podem efetuar remoção de nitrogênio, mas qual sistema pode fazê-lo a um custo menor. No presente artigo, apresentaram-se equações para a capacidade de remoção de nitrogênio das quatro variantes do lodo ativado que foram discutidas. Claramente, as quatro opções levam a resultados muito diferentes, como mostra a Fig. 15, onde se encontra o valor da capacidade de remoção de nitrogênio das diferentes alternativas. Os valores máximos de cada alternativa estão indicados. Essas diferenças não se devem à cinética intrínseca dos processos de nitrificação e desnitrificação: as mesmas equações básicas para descrever esses processos foram usadas nas quatro variantes consideradas. A diferença nos resultados se deve principalmente à diferença de sedimentabilidade de lodo em sistemas de fluxo contínuo e de operação em regime de bateladas sequenciais. Como a sedimentabilidade de lodo não está incorporada nos modelos de lodo ativado da IWA (HENZE et al, 1986 e 1994), essas implicações não se tornam explícitas.

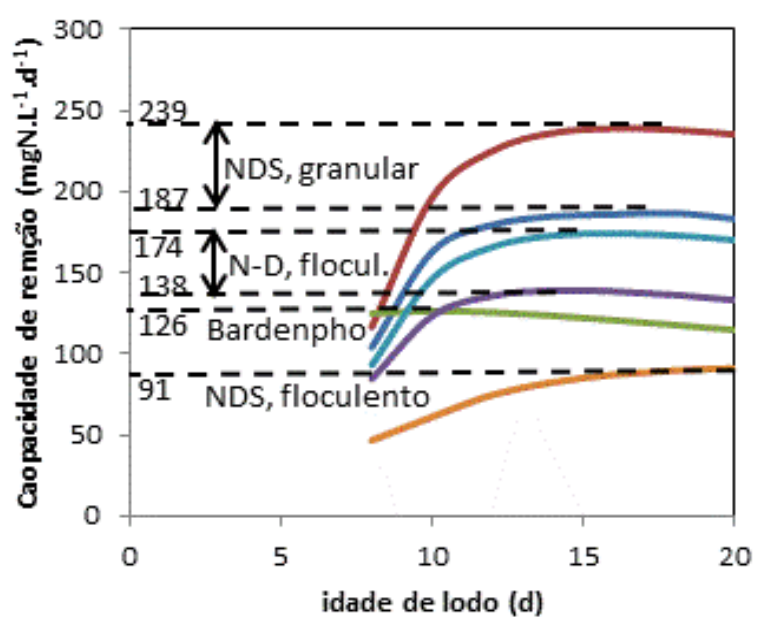

Figura 15: Capacidade de remoção de nitrogênio em diferentes sistemas de lodo ativado (condições de Tabela 1)

O sistema RBS com um reator unitário e lodo granular se destaca como a unidade com maior capacidade de remoção de nitrogênio. As razões para o desempenho superior do reator NDS com lodo granular são que (1) o lodo tem sedimentação excelente e pode ser operado com uma concentra- 
ção de lodo muito superior aos sistemas com lodo floculento e (2) que pode ser operado a uma alta concentração de OD (2 mg. $\mathrm{L}^{-1}$ na investigação) e ainda assim manter uma taxa de desnitrificação perto da máxima, obtida em condições anóxicas. O reator NDS, sendo um sistema de reator unitário único, tem a vantagem adicional de ser simples de operar. Os sensores podem garantir uma operação automatizado sob condições ótimas, em particular no que diz respeito à concentração de $O D$ ( $2 \mathrm{mg} \mathrm{L}^{-1}$ ). Sensores também podem ser usados para detectar o fim do período de aeração: o fim da nitrificação quando todo o amônio é oxidado resulta numa queda súbita na taxa de consumo de oxigênio, que pode ser detectada facilmente, por exemplo, com um respirômetro. Alternativamente, o fim do processo de nitrificação também significa que não mais acidez é gerada e, portanto, o pH irá parar para diminuir e pode mesmo subir um pouco, o que também pode ser utilizado para detectar o final do tratamento biológico de uma batelada. A operação de sistemas RBS também reduz os custos de operação quando a vazão é variável, o que quase sempre será o caso. Na prática, durante as primeiras seis horas do dia, a geração de esgoto é muito pequena, e no final da semana pode também ser muito mais baixa do que durante a semana. Quando a quantidade de esgoto a ser tratada é insuficiente para alimentar uma batelada ao reator, a alimentação pode simplesmente ser adiada até que o volume necessário para uma batelada se torne disponível, enquanto a aeração fica desligada. Nesse contexto, o sistema RBS é muito diferente dos sistemas de fluxo contínuo, em que a aeração não pode ser interrompida.

Até certo ponto, as diferentes configurações de lodo ativado influenciam as características do lodo gerado (coluna 3 na Tabela 1). Não há dúvida de que os sistemas RBS têm diferentes propriedades mecânicas: a sedimentabilidade de lodo RBS, como expressa em termos da equação Vesilind, é muito superior à dos sistemas de fluxo contínuo. Quanto às propriedades biológicas, as experiências têm mostrado que algumas das características são afetadas pelo tipo de sistema que é operado, enquanto outras não. De um modo geral, as constantes de velocidade do processo de nitrificação $\left(\mu_{\max } k_{n}, b_{n}\right.$ e $\left.k_{o}\right)$ e de desnitrificação $\left(k_{1}, k_{2}\right.$ e $\left.k_{3}\right)$ não são muito afetadas. No sistema Bardenpho, a constante $k$ varia usualmente desde $k=0,35-0,45$ L.g ${ }^{-1}$ e vo $=10$ a 15 m.h ${ }^{-1}$. Para sistemas RBS, valores típicos indicam muito melhor sedimentação: $k=0,15-0,25 \mathrm{~L} \cdot \mathrm{g}^{-1}$ e $\mathrm{v}_{\mathrm{o}}=12$ a 20 $\mathrm{m} \cdot \mathrm{h}^{-1}$, dependendo se o lodo é floculento ou granular. Essa diferença favorece sistemas RBS porque podem ser operados com maior concentração de lodo e, portanto, menor custo de investimento. Os sistemas RBS podem ser operados com uma concentração de lodo de 8 a $10 \mathrm{~g} \cdot \mathrm{L}^{-1}$, enquanto em sistemas de fluxo contínuo a concentração está na faixa de 3-5 g.L $\mathrm{L}^{-1}$. Na verdade, a sedimentação de lodo granular é tão boa que limitação da concentração de lodo é mais econômica do que operacional: a concentrações muito elevadas de lodo (> $\left.10 \mathrm{~g} \cdot \mathrm{L}^{-1}\right)$, a capacidade de aeração é fortemente reduzida pelo lodo mais espesso e aumenta muito o consumo de energia para aeração. Assim, essa opção é contraproducente, porque o aumento do custo operacional (aeração) não é compensado suficientemente por uma diminuição dos custos de investimento (volume).

$\mathrm{Na}$ investigação experimental, as constantes cinéticas para a nitrificação e desnitrificação de lodo granulado foram um pouco maiores que as constantes para lodo floculento. A constante de crescimento máximo das nitrificantes, $\mu_{\text {max }}$, foi cerca de $50 \%$ mais elevada no lodo granular $\left(0,52 \mathrm{~d}^{-1}\right)$, contra um valor de 0,30 a 0,35 $\mathrm{d}^{-1}$ muitas vezes encontrado para o lodo floculento gerado de esgoto de Campina Grande. A constante de desnitrificação, $k_{2}$, foi $20 \%$ maior do que o valor normalmente encontrado para lodo floculento $(0,18$, contra 0,15 $\left.\mathrm{mgN} \cdot \mathrm{mg}^{-1} \mathrm{X}_{\mathrm{a}} \cdot \mathrm{d}^{-1}\right)$. Não está claro se isso poderia ser atribuído ao fato de uma parte da massa do lodo heterotrófico ser composta de Organismos Acumuladores de Fosfato (PAO), que se tornou claro 
pela remoção de $\mathrm{P}$ de excesso que foi observada. PAOs são conhecidos por terem uma muito maior taxa de desnitrificação do que as bactérias comuns (CLAYTON et al, 1991). Todavia isso não explica o valor alto de $\mu_{\max }$ para lodo granulado.

Na prática, o sistema NDS com lodo floculento pode apresentar uma capacidade de remoção de nitrogênio bem mais elevada do que o valor que foi calculado no presente documento ( $91 \mathrm{mg} \cdot \mathrm{L}^{-1} \cdot \mathrm{d}^{-1}$ ), que se baseia nas observações de um reator em escala de bancada ( $6 \mathrm{~L}$ ) bem controlado. Isso pode ser atribuído ao fato de que, principalmente em grandes tanques de arejamento, é impossível ter uma concentração de OD uniforme e muito baixa. $\mathrm{Na}$ prática, portanto, a concentração não é uniforme, e há zonas com uma concentração relativamente elevada de OD (digamos cerca de $1 \mathrm{mg} \cdot \mathrm{L}^{-1}$ ) e zonas com uma concentração muito baixa $\left(<0,1 \mathrm{mg} \cdot \mathrm{L}^{-1}\right)$. Nessas zonas, as taxas de nitrificação e desnitrificação, respectivamente, estarão perto dos seus valores máximos. Nessas condições, o sistema começa a operar mais como um sistema N-D em vez de NDS, e a capacidade de remoção de nitrogênio aumenta correspondentemente. Conclui-se que, quando lodo floculento é usado em um reator NDS, é realmente uma vantagem se o controle da concentração de OD não for muito eficiente, de modo que a sua concentração não seja rigorosamente uniforme: zonas aeróbias e anóxicas vão aumentar a remoção de nitrogênio.

\section{CONCLUSÕES}

(1) Há dois fatores que favorecem a remoção biológica de nitrogênio pelos processos de nitrificação e desnitrificação em sistemas de lodo ativado no Brasil: (1) a temperatura alta do esgoto, que acelera as reações bioquímicas, e (2) a razão NTK/DQQO relativamente baixa, que caracteriza esgoto de países em desenvolvimento.
(2) Foram apresentados modelos para descrever a capacidade de remoção de nitrogênio em quatro variantes do sistema de lodo ativado. Três eram operados com lodo floculento: (1) o sistema Bardenpho, (2) o sistema RBS com reator unitário para Nitrificação e Desnitrificação Simultânea (NDS) e (3) o sistema RBS com dois reatores: um aeróbio e o outro anóxico (N-D). O quarto sistema era um RBS NDS com lodo granular.

(3) Mostrou-se que todos os sistemas têm potencial de produzir um efluente essencialmente livre de nitrogênio, mas a capacidade de remoção de nitrogênio por unidade de volume do sistema e por unidade de tempo depende fortemente da variante de lodo ativado utilizada.

(4) O sistema RBS com lodo granular tinha uma capacidade de remoção de nitrogênio maior que os outros. Essa grande capacidade se deve à possibilidade de manter numa configuração NDS com reator unitário uma concentração de OD elevada (2 mg.L-1) sem impacto grande sobre a capacidade de desnitrificação, podendo assim ser mantidas altas taxas de nitrificação e de desnitrificação durante o período de aeração.

(5) Mostrou-se que a capacidade de remoção de nitrogênio no sistema $N-D$ foi a maior dos sistemas de lodo floculento, enquanto o sistema NDS tinha a menor capacidade.

(6) Os sistemas RBS são favorecidos por uma maior sedimentabilidade de lodo que se desenvolve e que permite operação a uma concentração de lodo mais elevada e, portanto, um volume menor, reduzindo assim o custo de investimento.

(7) A concentração muito baixa necessária no sistema NDS com lodo floculento é muito difícil de manter na prática em sistemas com volumes grandes e operando a taxa variável de consumo de oxigênio, reduzindo, assim, mais ainda a capacidade de remoção de nitrogênio que já é baixa. 


\section{AGRADECIMENTOS}

Esta pesquisa foi realizada com o apoio do governo Brasileiro por meio da ANA (Agência Nacional das Águas) e do CNPq (Conselho Nacional de Pesquisa Tecnológica).

\section{REFERÊNCIAS}

BARNARD, J. L. Biological Denitrifaction. Water Pollution Control.v.72, n. 6, p. 705 720, 1976.

DE KREUK, M. K.; HEIJNEN, J. J. VAN LOOSDRECHT, M. C. M. (2005) Simultaneous COD, Nitrogen and Phosphate Removal by Aerobic Granular Sludge. Biotechnolology and Bioengineering. v. 90, n. (6) 761-769.

DE KREUK MC. Aerobic Granular Sludge Scaling up a new technology. 2006. Tese de PhD da Universidade Tecnológica de Delft, 2006.

DOWNING A.L., PAINTER H.A. E KNOWLES G. (1964); “Nitrification in the Activated Sludge Process"; J. Proc. Inst. Sew. Purif., vol. 64, no. 2, pp. 130158.

HELLINGA, C. et al.; The SHARON process: an innovative method for nitrogen removal from ammonia-rich wastewater. Water Science and Technology.v. 37, p. 135-142, 1998.

HENZE M. et al. "Activated sludge model No. 1". Scientific and Technical Reports. No 1, IAWPRC, London, U.K.; 1986.

HENZE M. et al.. "Activated sludge model No. 2"; IAWQ, London, U.K.; 1994

LUDZACK F.J. e ETTINGER M.G. (1962); “Controlling Operation to Minimize Activated Sludge Effluent Nitrogen”, J. Wat. Pollut. Control Fed., vol. 34, pp 920931.

MARAIS G.V.R.; EKAMA, G.A. The Activated Sludge Process: Steady State Behaviour. Water S.A.v. 2, n. 4, p. 163 200, 1976.

MATSCHE. The Elimination of Nitrogen in the Treatment Plant of Vienna Blumenthal. Water Research. v. 6, p. 485 486, 1972.
POCHANA, K. KELLER, J.; LANT, P. Model development for simultaneous nitrification and denitrification. Water Science and Technology. v. 39, n. 1, p. 235-243, 1999.

PRONK, M. et al.. Full scale performance of the aerobic granular sludge process for sewage treatment. Water Research. v. 1, n.84, p. 207-217, 2015.

RITTMANN, B. E; LANGELAND, W, E. Simultaneous denitrification with nitrification in single-channel oxidation ditches. Journal WPCF, v.57, n. 4, p. $300-308,1985$.

SATOH, H.; NAKAMURA, Y.; ONO, H.; OKABE, S. Effect of Oxygen Concentration on Nitrification and Denitrification in Single Activated Sludge Floes. Biotechnology and Bioengineering. v.83,n. 5, p. 604-607, 2003.

SIEBRITZ, I. P.; EKAMA, G. A.; MARAIS, G.V. R. A parametric model for biological excess phosphorus removal. Water Science Technology. v. 15, n. 3/4, p. 127-52, 1982.

STENSTRÖM, M. K.; PODUSKA, R. A. The Effect of Dissolved Oxygen Concentration on Nitrification. Water Research. v. 14, n. 6, p. $645650,1980$.

VAN HAANDEL, A. C.; EKAMA, G. A.; MARAIS, G. v R. The activated sludge process single sludge denitrification. Water Research, $v$. 15, n. 10, p. $1135-1152,1981$.

VAN HAANDEL A. C. E MARAIS G.v.R. (1999): O Comportamento do sistema de lodo ativado: Teoria e aplicações para operação e projetos, Campina Grande: Editora Epgraf.

VAN HAANDEL A.C. KATO M.T. E VON SPERLING M. (2009): Remoção Biológica de Nitrogênio: Aplicações para o Sistema de Lodo Ativado. In: MOTA F.S.B e VON SPERLING M (Eds.) Nutrientes de esgoto sanitários: utilização e remoção. Rio de Janeiro: $A B E S$. ISBN: 978-85-7022-164-3.

VESILIND, P. A. Theoretical considerations: Design of prototype thickeners from batch settling tests. Water Sewage Works, v. 115, n. 7, p. $302-307,1968$.

WUHRMANN K. (1964); Remoção de nitrogênio em sistemas de tratamento de águas residuárias” (em alemão). Verh. Int. Ver. Theor. Angew. Limnol., vol. 15. 